\title{
19. HIGH RESOLUTION CARBONATE RECORDS FROM THE HYDRAULIC PISTON CORED SECTION OF SITE $572^{1}$
}

\author{
Nicklas G. Pisias, College of Oceanography, Oregon State University \\ and \\ Warren L. Prell, Department of Geological Sciences, Brown University ${ }^{2}$
}

\begin{abstract}
At Site 572 , located at $1^{\circ} \mathrm{N}, 114^{\circ} \mathrm{W}(3903 \mathrm{~m}$ water depth), we recovered a continuous hydraulic piston cored section of upper Miocene to upper Pleistocene pelagic sediments. The sediment is composed of biogenic carbonate and silica with nonbiogenic material as a minor component. Detailed analysis of the calcium carbonate content shows that the degree of variability in carbonate deposition apparently changed markedly between the late Miocene and Pliocene at this equatorial Pacific site. During this interval carbonate mass accumulation rates decreased from 2.6 to $0.8 \mathrm{~g} / \mathrm{cm}^{2}$ per $10^{3}$ yr. If we assume that variations in $\mathrm{CaCO}_{3}$ content reflect changes in the degree of dissolution, then the detailed carbonate analysis would suggest that the degree of variability in carbonate deposition decreases by a factor of 5 as the dominant wavelength of variations increases significantly. However, if the variability in carbonate concentration is described in terms of changes in mean mass accumulation, calculations then suggest that relatively small changes in noncarbonate rates may be important in controlling the observed carbonate records. In addition, the analysis suggests that the degree of variability observed in pelagic carbonate data may in part reflect total accumulation rates. Intervals with high sedimentation rates show lower amplitude variations in concentration than intervals with lower sedimentation rates for the same degree of change in the carbonate accumulation rate.
\end{abstract}

\section{INTRODUCTION}

Variations in calcium carbonate content have been used by marine geologists and paleoceanographers as a tool for stratigraphic correlation (Prell, 1978; Dunn and Moore, 1981; Hays et al., 1969) and as an indicator of oceanic response to global climate change. In general, the resolution attained in studies of calcium carbonate deposition reflects the quality and sedimentation rate of the sediment sections available.

Studies of Pleistocene sediments typically attain sampling resolution on the order of a few thousand years, and stratigraphic resolution on the order of $50,000 \mathrm{yr}$. is possible, as shown by Hays et al. (1969). These studies used samples of undisturbed sediment collected by standard piston coring techniques. The availability of undisturbed sections of pre-Pleistocene sediments was severely limited until the development of the hydraulic piston coring capability on the Glomar Challenger. Thus, most studies of carbonate variation in pre-Pleistocene sediments have used samples from either rotary drilled sections or the few piston cores that sampled areas where much of the Pleistocene section had been removed by erosion. Studies of carbonate variations in pre-Pleistocene sections (e.g., Dunn and Moore, 1981; Vincent, 1981) typically have sample spacing of several tens of thousands of years and demonstrate stratigraphic resolution on the order of 100,000 to 500,000 yr. A few studies have examined carbonate variations in pre-Pleisto-

\footnotetext{
${ }^{1}$ Mayer, L., Theyer, F., et al., Init. Repts. DSDP, 85: Washington (U.S. Govt. Printing Office).

Addresses: (Pisias) College of Oceanography, Oregon State University, Corvallis, OR 97331; (Prell) Department of Geological Sciences, Brown University, Providence, RI 02901.
}

cene sediments at a time resolution comparable to that in Pleistocene studies (Dean et al., 1981; Gardner, 1982; Moore et al., 1982), but the coverage in time and space is still extremely limited.

Because of the limited resolution of available Cenozoic carbonate records, it has been difficult to evaluate whether the ocean's carbonate system has displayed the same high degree of variability in the more remote geologic past as that observed in the last $1 \mathrm{~m}$.y. Detailed stable isotope records from the Miocene and Pliocene (Woodruff et al., 1981; Pisias and Shackleton, this volume; Prell, 1982 and this volume) suggest a high degree of climatic variability and lead us to expect variations in carbonate sedimentation as well. Detailed sampling was completed in a number of cores from Site 572 to examine the variability of carbonate deposition in the equatorial Pacific. In this report we present high resolution carbonate analyses from the hydraulic piston cored section of Site 572.

\section{SAMPLING STRATEGY AND METHODS}

At Site $572\left(01^{\circ} 26.09^{\prime} \mathrm{N}, 113^{\circ} 50.52^{\prime} \mathrm{W}\right.$, water depth, $3903 \mathrm{~m}$ ), the hydraulic piston corer (HPC) recovered sediments to $154 \mathrm{~m}$ sub-bottom and provided a high quality, undisturbed, sediment section spanning the last $6 \mathrm{~m} . \mathrm{y}$. During this period Site $\mathbf{5 7 2}$ has always been within the equatorial divergence zone. Hence, sedimentation rates have been high throughout much of its history, with sedimentation rates of 13 to $21 \mathrm{~m} / \mathrm{m} . \mathrm{y}$. in the upper $68 \mathrm{~m}$ increasing to $50 \mathrm{~m} / \mathrm{m}$.y. between 68 and $208 \mathrm{~m}$ sub-bottom (Table 1).

Selected intervals were sampled at $10-\mathrm{cm}$ spacing to examine the sedimentary response to oceanographic and climatic variability in the latest Miocene and Pliocene in 
Table 1. Sedimentation and mass accumulation rate estimates for the HPC section of Site 572 .

\begin{tabular}{ccccccc}
\hline $\begin{array}{c}\text { Sub-bottom } \\
\text { depth } \\
(\mathrm{m})\end{array}$ & $\begin{array}{c}\text { Age } \\
(\mathrm{Ma})\end{array}$ & $\begin{array}{c}\text { Rate } \\
(\mathrm{m} / \mathrm{m} . \mathrm{y} .)\end{array}$ & $\begin{array}{c}\text { Mean } \\
\text { density } \\
\left(\mathrm{g} / \mathrm{cm}^{3}\right)\end{array}$ & $\begin{array}{c}\text { Mean } \\
\mathrm{CaCO}_{3} \\
(\%)\end{array}$ & $\begin{array}{c}\text { Mass accumulation } \\
\text { rate }\left(\mathrm{g} / \mathrm{cm}^{2}\right. \\
\left.\text { per } 10^{3} \mathrm{yr} .\right)\end{array}$ \\
\hline 9.5 & 0.55 & 13 & 0.57 & 79 & 0.58 & 0.16 \\
29.0 & 2.0 & 21 & 0.61 & 73 & 0.93 & 0.35 \\
49.0 & 2.95 & 15.2 & 0.70 & 75 & 0.80 & 0.20 \\
68.0 & 4.2 & 50.0 & 0.72 & 73 & 2.60 & 1.00 \\
208.0 & 6.95 & & & & & \\
\hline
\end{tabular}

detail. The intervals were selected on the basis of four criteria: (1) high sedimentation rates (so that high resolution records could be obtained); (2) shipboard evaluation of carbonate fossil preservation (to ensure adequate numbers of foraminiferal tests for later stable isotopic analyses); (3) intervals that spanned major paleoclimatic or paleoceanographic events; and (4) at least $15 \mathrm{~m}$ of continuous good recovery so that a long enough sequence could be sampled for detailed time and frequency domain statistical analysis.

Three intervals from Site 572 were sampled in detail: Cores $572 \mathrm{C}-4$ to $572 \mathrm{C}-7,29$ to $68 \mathrm{~m}$ sub-bottom; Cores $572 \mathrm{~A}-11$ and 12,92 to $110 \mathrm{~m}$ sub-bottom; and Cores $572 \mathrm{C}-15$ and 16,121 to $134 \mathrm{~m}$ sub-bottom. The first sample interval represents the time interval from 2 to 4.6 Ma and spans the time when major glaciation supposedly began in the Northern Hemisphere (Shackleton et al., 1984). A detailed stable isotopic analysis of this section is presented by Prell (this volume). The sedimentation rate in this interval is about $15 \mathrm{~m} / \mathrm{m}$.y. so that the sampling resolution is on the order of $6000 \mathrm{yr}$.

The sedimentation rate below $68 \mathrm{~m}$ sub-bottom is estimated to be $\sim 50 \mathrm{~m} / \mathrm{m}$.y. Thus, the intervals from 92 to $110 \mathrm{~m}$ and 121 to $134 \mathrm{~m}$ sub-bottom provided very high resolution records of carbonate variations in the latest Miocene. These intervals sample the time periods of about 4.6 to $5 \mathrm{Ma}$ and 5.2 to $5.3 \mathrm{Ma}$ with a sample resolution of $2000 \mathrm{yr}$. Stable isotopic analyses for these intervals are in progress.

Analyses of calcium carbonate in samples from 29 to $68 \mathrm{~m}$ sub-bottom were completed on paired samples for isotopic analysis by using a modified version of the Jones and Kaiteris (1983) vacuum-gasometric technique. In the system used for this study, reactions are measured at atmospheric pressure and measurements are made with a differential pressure gauge. Analytic precision is estimated to be $\sim 0.5 \%$ from replicate analyses. Calcium carbonate was determined in the interval from 92 to $134 \mathrm{~m}$ sub-bottom using the carbonate bomb technique as modified by Dunn (1980). Analytic precision based on homogenized sample splits is $1 \% \mathrm{CaCO}_{3}$, and on samples split without homogenization it is $2 \% \mathrm{CaCO}_{3}$. All carbonate analyses are listed in Table 2 and plotted as a function of sub-bottom depth in Figures 1 and 2 .

\section{RESULTS AND DISCUSSION}

\section{Sample Aliasing}

Included in Figures 1 and 2 are the carbonate data obtained on board the Glomar Challenger. Shipboard analyses were made using the carbonate bomb technique. In general, two samples per core section (at about $75 \mathrm{~cm}$ sample spacing) were analyzed on board ship. All shipboard analyses shown are from Hole 572A. Therefore, only in the interval from 92 to $110 \mathrm{~m}$ sub-bottom are the high resolution samples from the same cores as the shipboard data. Where data from the high resolution studies come from the same hole (572A) as the shipboard data, agreement is very good (see Fig. 3B). If we assume that sub-bottom depths are calculated correctly, then differences between the data sets from two different holes, as over the interval from 29 to $68 \mathrm{~m}$, reflect inhomogeneity in the horizontal distribution of calcium carbonate within the distance between the different holes at Site 572 . More important, this can also reflect the effect of sample aliasing that results from the marked difference in resolution of the two data sets.

Aliasing, as applied to sampling in the time domain, (or in the depth domain, as with deep-sea sediments), results from sampling at a resolution too low for the wavelength of variation. In effect, short-wavelength components that are sampled at long sampling intervals appear as long-wavelength variations. For example, in Figure $3 \mathrm{~A}$ a hypothetical carbonate record with a wavelength of $1.25 \mathrm{~m}$ is shown. If the core is sampled at $145-\mathrm{cm}$ intervals, the resulting data look like a long-period component of $11 \mathrm{~m}$. Thus, the true information in the data set is "aliased" as a signal of much lower frequency. Note that in this example the true amplitude of variations can be determined from the aliased data.

An example of aliasing from the Site $\mathbf{5 7 2}$ data is shown in Figure 3B. The shipboard data at $75-\mathrm{cm}$ intervals would suggest an interval of relatively constant $( \pm 5 \%)$ $\mathrm{CaCO}_{3}$ content between 100 and $110 \mathrm{~m}$ sub-bottom. The detailed sampling, however, shows variations on the order of $\pm 10 \% \mathrm{CaCO}_{3}$. The low-resolution sampling aliased variability of $15 \% \mathrm{CaCO}_{3}$ with a wavelength of $1 \mathrm{cycle} / \mathrm{m}$ to a constant signal of very low frequency. In this case, the 15,000 -yr. sampling represented by the shipboard analyses lost essentially all the information about the $\mathrm{CaCO}_{3}$ variations, not even recovering the amplitude of variation.

The effect of aliasing on a data set cannot be determined from that data set. Information not contained in the data set is needed to evaluate aliasing, and only highresolution sampling can reveal the effects of short-wavelength components on the aliased "low frequencies."

\section{Depth Versus Time Domain}

The data from Site 572 span almost 4 m.y., from the latest Miocene through most of the Pliocene. In general, the depth series are very similar in their general appearance and statistical properties (Table 3; Figs. 1 and 2). The mean values for four intervals range from 69 to 
Table 2. $\mathrm{CaCO}_{3}$ percentages in all samples analyzed at Site 572 .

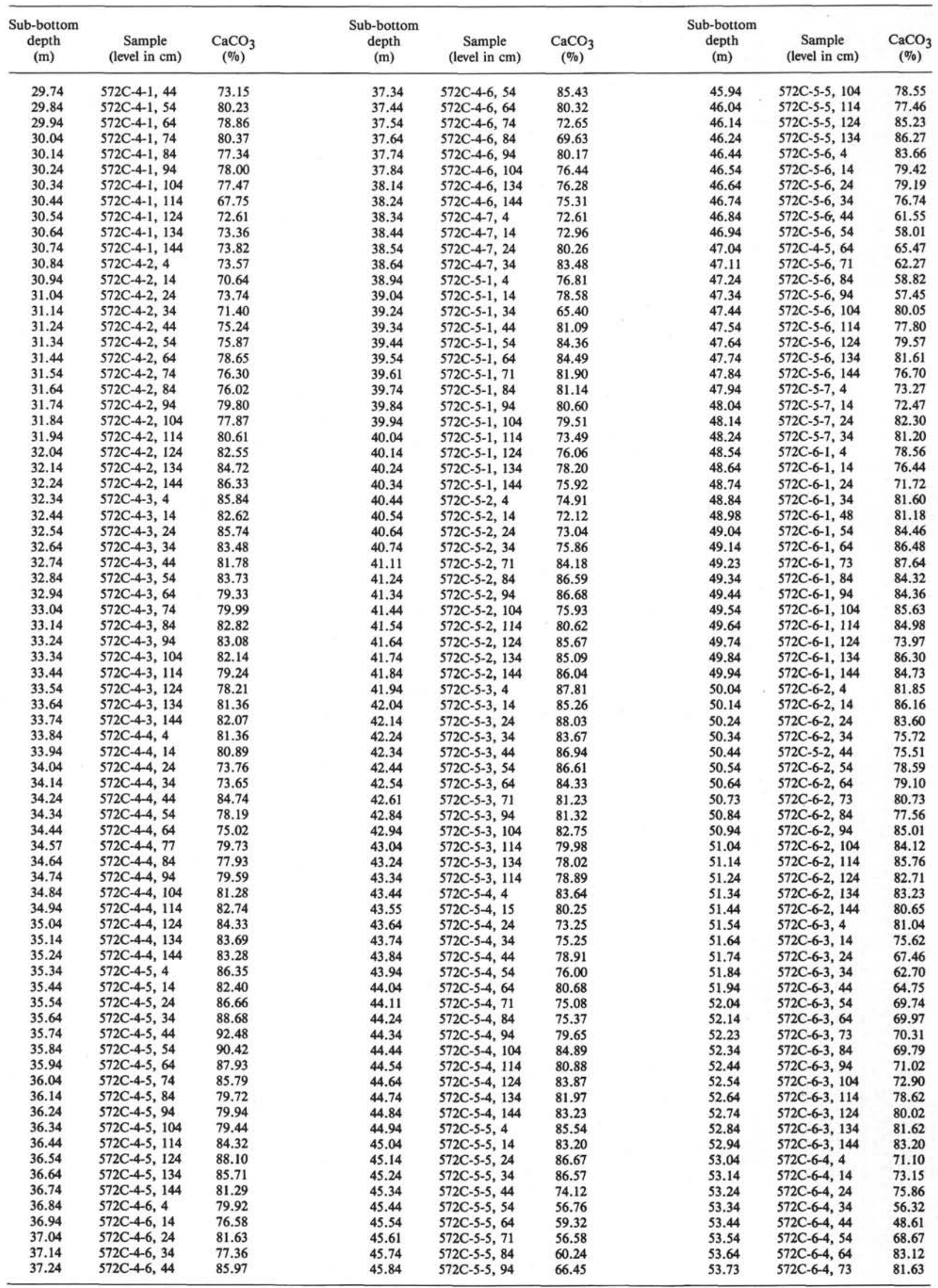


N. G. PISIAS, W. L. PRELL

Table 2. (Continued).

\begin{tabular}{|c|c|c|c|c|c|c|c|c|}
\hline $\begin{array}{l}\text { Sub-bottom } \\
\text { depth } \\
\text { (m) }\end{array}$ & $\begin{array}{c}\text { Sample } \\
\text { (level in cm) }\end{array}$ & $\begin{array}{c}\mathrm{CaCO}_{3} \\
(\%)\end{array}$ & $\begin{array}{l}\text { Sub-bottom } \\
\text { depth } \\
\text { (m) }\end{array}$ & $\begin{array}{c}\text { Sample } \\
\text { (level in } \mathrm{cm} \text { ) }\end{array}$ & $\begin{array}{c}\mathrm{CaCO}_{3} \\
(\%)\end{array}$ & $\begin{array}{l}\text { Sub-bottom } \\
\text { depth } \\
\text { (m) }\end{array}$ & $\begin{array}{c}\text { Sample } \\
\text { (level in } \mathrm{cm} \text { ) }\end{array}$ & $\begin{array}{c}\mathrm{CaCO}_{3} \\
(\%)\end{array}$ \\
\hline 53.84 & $572 C-6-4,84$ & 76.66 & 61.74 & $572 C-7-3,64$ & 86.54 & 93.43 & $572 \mathrm{~A}-11-2,53$ & 80.04 \\
\hline 53.94 & $572 \mathrm{C}-6-4,94$ & 74.44 & 61.84 & $572 \mathrm{C}-7-3,74$ & 87.74 & 93.53 & $572 \mathrm{~A}-11-2,63$ & 83.73 \\
\hline 54.04 & $572 C-6-4,104$ & 64.87 & 61.94 & $572 \mathrm{C}-7-3,84$ & 87.32 & 93.59 & $572 A-11-2,69$ & 82.97 \\
\hline 54.14 & $572 \mathrm{C}-6-4,114$ & 60.10 & 62.04 & $572 \mathrm{C}-7-3,94$ & 87.59 & 93.73 & $572 \mathrm{~A}-11-2,83$ & 78.30 \\
\hline 54.34 & $572 C-6-4,134$ & 83.70 & 62.14 & $572 C-7-3,104$ & 90.36 & 93.83 & $572 \mathrm{~A}-11-2,93$ & 76.24 \\
\hline 54.44 & $572 \mathrm{C}-6-4,144$ & 88.52 & 62.24 & $572 C-7-3,114$ & 89.72 & 93.93 & $572 \mathrm{~A}-11-2,103$ & 75.59 \\
\hline 54.54 & $572 C-6-5,4$ & 88.58 & 62.34 & $572 \mathrm{C}-7-3,124$ & 86.40 & 94.03 & $572 \mathrm{~A}-11-2,113$ & 72.88 \\
\hline 54.64 & $572 C-6-5,14$ & 90.37 & 62.44 & $572 C-7-3,134$ & 84.43 & 94.13 & $572 \mathrm{~A}-11-2,123$ & 72.88 \\
\hline 54.74 & $572 \mathrm{C}-6-5,24$ & 86.54 & 62.54 & $572 C-7-3,144$ & 89.43 & 94.23 & $572 \mathrm{~A}-11-2,133$ & 71.47 \\
\hline 54.84 & $572 \mathrm{C}-6-5,34$ & 78.96 & 62.64 & $572 C-7-4,4$ & 87.35 & 94.33 & $572 \mathrm{~A}-11-2,143$ & 68.22 \\
\hline 54.94 & $572 C-6-5,44$ & 76.83 & 62.74 & $572 \mathrm{C}-7-4,14$ & 83.00 & 94.43 & $572 \mathrm{~A}-11-3,3$ & 65.93 \\
\hline 55.04 & $572 \mathrm{C}-6-5,54$ & 84.19 & 62.84 & $572 \mathrm{C}-7-4,24$ & 84.66 & 94.53 & $572 \mathrm{~A}-11-3,13$ & 64.31 \\
\hline 55.14 & $572 C-6-5,64$ & 83.70 & 62.94 & $572 \mathrm{C}-7-4,34$ & 85.85 & 94.63 & $572 \mathrm{~A}-11-3,23$ & 70.23 \\
\hline 55.23 & $572 \mathrm{C}-6-5,73$ & 86.88 & 63.04 & $572 \mathrm{C}-7-4,44$ & 75.57 & 94.73 & $572 \mathrm{~A}-11-3,33$ & 67.54 \\
\hline 55.34 & $572 \mathrm{C}-6-5,84$ & 86.41 & 63.14 & $572 \mathrm{C}-7-4,54$ & 72.67 & 94.83 & $572 A-11-3,43$ & 67.00 \\
\hline 55.44 & $572 \mathrm{C}-6-5,94$ & 79.89 & 63.24 & $572 \mathrm{C}-7-4,64$ & 85.92 & 94.93 & $572 A-11-3,53$ & 76.15 \\
\hline 55.54 & $572 C-6-5,104$ & 70.20 & 63.33 & $572 C-7-4,73$ & 85.99 & 95.03 & $572 \mathrm{~A}-11-3,63$ & 70.23 \\
\hline 55.64 & $572 C-6-5,114$ & 76.02 & 63.44 & $572 \mathrm{C}-7-4,84$ & 86.90 & 95.10 & $572 \mathrm{~A}-11-3,70$ & 71.63 \\
\hline 55.74 & $572 \mathrm{C}-6-5,124$ & 75.41 & 63.54 & $572 \mathrm{C}-7-4,94$ & 88.01 & 95.23 & $572 \mathrm{~A}-11-3,83$ & 72.93 \\
\hline 55.84 & $572 C-6-5,134$ & 75.98 & 63.64 & $572 C-7-4,104$ & 88.34 & 95.33 & $572 \mathrm{~A}-11-3,93$ & 75.07 \\
\hline 55.94 & $572 C-6-5,144$ & 75.25 & 63.74 & $572 C-7-4,114$ & 88.89 & 95.43 & $572 \mathrm{~A}-11-3,103$ & 76.15 \\
\hline 56.04 & $572 C-6-6,4$ & 72.89 & 63.84 & $572 C-7-4,124$ & 86.28 & 95.53 & $572 \mathrm{~A}-11-3,113$ & 74.32 \\
\hline 56.14 & $572 C-6-6,14$ & 75.76 & 63.94 & $572 \mathrm{C}-7-4,134$ & 90.39 & 95.63 & $572 \mathrm{~A}-11-3,123$ & 73.46 \\
\hline 56.24 & $572 C-6-6,24$ & 73.00 & 64.14 & $572 C-7-5,4$ & 86.16 & 95.73 & $572 \mathrm{~A}-11-3,133$ & 72.92 \\
\hline 56.34 & $572 \mathrm{C}-6-6,34$ & 74.20 & 64.24 & $572 \mathrm{C}-7-5,14$ & 73.01 & 95.83 & $572 \mathrm{~A}-11-3,143$ & 68.08 \\
\hline 56.44 & $572 C-6-6,44$ & 83.82 & 64.34 & $572 \mathrm{C}-7-5,24$ & 73.35 & 96.03 & $572 A-11-4,13$ & 70.77 \\
\hline 56.54 & $572 \mathrm{C}-6-6,54$ & 83.27 & 64.44 & $572 \mathrm{C}-7-5,34$ & 70.95 & 96.13 & $572 \mathrm{~A}-11-4,23$ & 71.63 \\
\hline 56.64 & $572 C-6-6,64$ & 76.97 & 64.54 & $572 \mathrm{C}-7-5,44$ & 80.02 & 96.23 & $572 \mathrm{~A}-11-4,33$ & 71.63 \\
\hline 56.73 & $572 \mathrm{C}-6-6,73$ & 61.27 & 64.64 & $572 \mathrm{C}-7-5,54$ & 85.36 & 96.33 & $572 \mathrm{~A}-11-4,43$ & 64.31 \\
\hline 56.84 & $572 \mathrm{C}-6-6,84$ & 59.84 & 64.74 & $572 C-7-5,64$ & 85.90 & 96.43 & $572 \mathrm{~A}-11-4,53$ & 68.08 \\
\hline 56.94 & $572 C-6-6,94$ & 59.60 & 64.83 & $572 \mathrm{C}-7-5,73$ & 80.14 & 96.53 & $572 A-11-4,63$ & 68.94 \\
\hline 57.04 & $572 C-6-6,104$ & 68.19 & 64.94 & $572 \mathrm{C}-7-5,84$ & 71.53 & 96.61 & $572 \mathrm{~A}-11-4,71$ & 72.38 \\
\hline 57.14 & $572 C-6-6,114$ & 71.46 & 65.04 & $572 \mathrm{C}-7-5,94$ & 85.74 & 96.73 & $572 \mathrm{~A}-11-4,83$ & 75.07 \\
\hline 57.24 & $572 \mathrm{C}-6-6,124$ & 67.22 & 65.14 & $572 C-7-5,104$ & 84.73 & 96.83 & $572 \mathrm{~A}-11-4,93$ & 75.61 \\
\hline 57.34 & $572 \mathrm{C}-6-6,134$ & 32.20 & 65.24 & $572 C-7-5,114$ & 84.52 & 96.93 & $572 A-11-4,103$ & 72.38 \\
\hline 57.44 & $572 \mathrm{C}-6-6,144$ & 66.97 & 65.34 & $572 C-7-5,124$ & 90.72 & 97.03 & $572 A-11-4,113$ & 68.94 \\
\hline 57.54 & $572 C-6-7,4$ & 80.19 & 65.44 & $572 C-7-5,134$ & 89.09 & 97.13 & $572 \mathrm{~A}-11-4,123$ & 70.77 \\
\hline 57.64 & $572 \mathrm{C}-6-7,14$ & 75.83 & 65.54 & $572 C-7-5,144$ & 89.66 & 97.23 & $572 \mathrm{~A}-11-4,133$ & 66.25 \\
\hline 57.74 & $572 \mathrm{C}-6-7,24$ & 79.79 & 65.64 & $572 C-7-6,4$ & 89.15 & 97.33 & $572 A-11-4,143$ & 65.93 \\
\hline 57.84 & $572 \mathrm{C}-6-7,34$ & 79.47 & 65.74 & $572 C-7-6,14$ & 83.93 & 97.53 & $572 \mathrm{~A}-11-5,13$ & 87.43 \\
\hline 58.14 & $572 \mathrm{C}-7-1,4$ & 65.13 & 65.84 & $572 C-7-6,24$ & 89.56 & 97.63 & $572 \mathrm{~A}-11-5,23$ & 67.54 \\
\hline 58.24 & $572 \mathrm{C}-7-1,14$ & 58.22 & 65.94 & $572 \mathrm{C}-7-6,34$ & 88.67 & 97.73 & $572 \mathrm{~A}-11-5,33$ & 99.99 \\
\hline 58.34 & $572 C-7-1,24$ & 54.18 & 66.04 & $572 \mathrm{C}-7-6,44$ & 89.80 & 97.83 & $572 \mathrm{~A}-11-5,43$ & 94.06 \\
\hline 58.44 & $572 C-7-1,34$ & 63.96 & 66.14 & $572 C-7-6,54$ & 89.68 & 97.93 & $572 \mathrm{~A}-11-5,53$ & 75.05 \\
\hline 58.54 & $572 C-7-1,44$ & 68.06 & 66.24 & $572 C-7-6,64$ & 91.28 & 98.03 & $572 A-11-5,63$ & 71.41 \\
\hline 58.64 & $572 C-7-1,54$ & 66.64 & 66.35 & $572 \mathrm{C}-7-6,75$ & 88.34 & 98.11 & $572 \mathrm{~A}-11-5,71$ & 68.64 \\
\hline 58.74 & $572 C-7-1,64$ & 68.82 & 66.44 & $572 \mathrm{C}-7-6,84$ & 86.74 & 98.23 & $572 \mathrm{~A}-11-5,83$ & 78.04 \\
\hline 58.83 & $572 \mathrm{C}-7-1,73$ & 74.74 & 66.54 & $572 \mathrm{C}-7-6,94$ & 83.11 & 98.33 & $572 \mathrm{~A}-11-5,93$ & 85.22 \\
\hline 58.94 & $572 C-7-1,84$ & 78.41 & 66.64 & $572 \mathrm{C}-7-6,104$ & 79.28 & 98.43 & $572 \mathrm{~A}-11-5,103$ & 83.01 \\
\hline 59.04 & $572 \mathrm{C}-7-1,94$ & 77.53 & 66.74 & $572 C-7-6,114$ & 74.16 & 98.53 & $572 \mathrm{~A}-11-5,113$ & 62.02 \\
\hline 59.14 & $572 C-7-1,104$ & 75.39 & 66.84 & $572 C-7-6,124$ & 73.93 & 98.63 & $572 \mathrm{~A}-11-5,123$ & 68.09 \\
\hline 59.24 & $572 C-7-1,114$ & 76.17 & 66.94 & $572 C-7-6,134$ & 70.40 & 98.73 & $572 \mathrm{~A}-11-5,133$ & 68.09 \\
\hline 59.34 & $572 \mathrm{C}-7-1,124$ & 71.62 & 67.04 & $572 C-7-6,144$ & 69.74 & 98.83 & $572 \mathrm{~A}-11-5,143$ & 64.10 \\
\hline 59.44 & $572 C-7-1,134$ & 74.31 & 67.14 & $572 C-7-7,4$ & 68.68 & 98.93 & $572 \mathrm{~A}-11-6,3$ & 40.87 \\
\hline 59.54 & $572 C-7-1,144$ & 71.94 & 67.24 & $572 \mathrm{C}-7-7,14$ & 76.01 & 99.03 & $572 A-11-6,13$ & 38.11 \\
\hline 59.64 & $572 C-7-2,4$ & 68.83 & 67.34 & $572 \mathrm{C}-7-7,24$ & 80.79 & 99.13 & $572 A-11-6,23$ & 51.94 \\
\hline 59.74 & $572 \mathrm{C}-7-2,14$ & 66.92 & 67.44 & $572 \mathrm{C}-7-7,34$ & 81.67 & 99.33 & $572 A-11-6,43$ & 44.75 \\
\hline 59.84 & $572 \mathrm{C}-7-2,24$ & 63.52 & & & & 99.53 & $572 A-11-6,63$ & 67.42 \\
\hline 59.94 & $572 \mathrm{C}-7-2,34$ & 64.07 & 91.53 & $572 \mathrm{~A}-11-1,13$ & 73.73 & 99.59 & $572 A-11-6,69$ & 66.32 \\
\hline 60.04 & $572 C-7-2,44$ & 76.75 & 91.73 & $572 \mathrm{~A}-11-1,33$ & 70.52 & 99.73 & $572 A-11-6,83$ & 47.51 \\
\hline 60.14 & $572 \mathrm{C}-7-2,54$ & 77.43 & 91.83 & $572 A-11-1,43$ & 74.81 & 100.03 & $572 A-11-6,113$ & 76.83 \\
\hline 60.24 & $572 \mathrm{C}-7-2,64$ & 76.35 & 91.93 & $572 \mathrm{~A}-11-1,53$ & 68.37 & 100.13 & $572 \mathrm{~A}-11-6,123$ & 74.06 \\
\hline 60.33 & $572 C-7-2,73$ & 77.97 & 92.03 & $572 \mathrm{~A}-11-1,63$ & 69.44 & 100.23 & $572 \mathrm{~A}-11-6,133$ & 74.95 \\
\hline 60.44 & $572 C-7-2,84$ & 78.42 & 92.11 & $572 \mathrm{~A}-11-1,71$ & 69.23 & 100.33 & $572 A-11-6,143$ & 77.38 \\
\hline 60.54 & $572 C-7-2,94$ & 88.16 & 92.23 & $572 \mathrm{~A}-11-1,83$ & 67.83 & 100.43 & $572 A-11-7,3$ & 75.72 \\
\hline 60.64 & $572 C-7-2,104$ & 86.16 & 92.33 & $572 \mathrm{~A}-11-1,93$ & 66.55 & 100.73 & $572 \mathrm{~A}-12-1,3$ & 55.13 \\
\hline 60.74 & $572 C-7-2,114$ & 85.80 & 92.43 & $572 \mathrm{~A}-11-1,103$ & 67.30 & 100.73 & $572 \mathrm{~A}-12-1,3$ & 45.85 \\
\hline 60.84 & $572 C-7-2,124$ & 84.69 & 92.53 & $572 \mathrm{~A}-11-1,113$ & 61.18 & 100.83 & $572 \mathrm{~A}-12-1,13$ & 52.91 \\
\hline 60.94 & $572 C-7-2,134$ & 85.00 & 92.63 & $572 \mathrm{~A}-11-1,123$ & 57.64 & 100.93 & $572 \mathrm{~A}-12-1,23$ & 57.34 \\
\hline 61.04 & $572 C-7-2,144$ & 88.71 & 92.73 & $572 \mathrm{~A}-11-1,133$ & 52.82 & 101.03 & $572 \mathrm{~A}-12-1,33$ & 66.21 \\
\hline 61.14 & $572 \mathrm{C}-7-3,4$ & 84.13 & 92.83 & $572 \mathrm{~A}-11-1,143$ & 65.15 & 101.03 & $572 A-12-1,33$ & 41.43 \\
\hline 61.24 & $572 \mathrm{C}-7-3,14$ & 85.07 & 92.93 & $572 \mathrm{~A}-11-2,3$ & 75.27 & 101.13 & $572 \mathrm{~A}-12-1,43$ & 72.86 \\
\hline 61.34 & $572 \mathrm{C}-7-3,24$ & 86.44 & 93.03 & $572 \mathrm{~A}-11-2,13$ & 68.76 & 101.23 & $572 \mathrm{~A}-12-1,53$ & 75.08 \\
\hline 61.44 & $572 \mathrm{C}-7-3,34$ & 83.81 & 93.13 & $572 \mathrm{~A}-11-2,23$ & 75.81 & 101.33 & $572 \mathrm{~A}-12-1,63$ & 69.53 \\
\hline 61.54 & $572 \mathrm{C}-7-3,44$ & 84.60 & 93.23 & $572 \mathrm{~A}-11-2,33$ & 77.33 & 101.33 & $572 A-12-1,63$ & 75.72 \\
\hline 61.64 & $572 \mathrm{C}-7-3,54$ & 86.24 & 93.33 & $572 A-11-2,43$ & 82.32 & 101.40 & $572 \mathrm{~A}-12-1,70$ & 70.64 \\
\hline
\end{tabular}


Table 2. (Continued).

\begin{tabular}{|c|c|c|c|c|c|c|c|c|}
\hline $\begin{array}{l}\text { Sub-bottom } \\
\text { depth } \\
\text { (m) }\end{array}$ & $\begin{array}{c}\text { Sample } \\
\text { (level in } \mathrm{cm} \text { ) }\end{array}$ & $\begin{array}{c}\mathrm{CaCO}_{3} \\
(\%)\end{array}$ & $\begin{array}{l}\text { Sub-bottom } \\
\text { depth } \\
\text { (m) }\end{array}$ & $\begin{array}{c}\text { Sample } \\
\text { (level in cm) }\end{array}$ & $\begin{array}{c}\mathrm{CaCO}_{3} \\
(\%)\end{array}$ & $\begin{array}{l}\text { Sub-bottom } \\
\text { depth } \\
\text { (m) }\end{array}$ & $\begin{array}{c}\text { Sample } \\
\text { (level in } \mathrm{cm} \text { ) }\end{array}$ & $\begin{array}{c}\mathrm{CaCO}_{3} \\
(\%)\end{array}$ \\
\hline 101.43 & $572 A-12-1,73$ & 74.95 & 108.83 & $572 A-12-6,63$ & 77.38 & 127.21 & $572 \mathrm{C}-15-5,11$ & 79.39 \\
\hline 101.53 & $572 \mathrm{~A}-12-1,83$ & 73.19 & 108.90 & $572 \mathrm{~A}-12-6,70$ & 74.35 & 127.30 & $572 \mathrm{C}-15-5,20$ & 80.55 \\
\hline 101.63 & $572 \mathrm{~A}-12-1,93$ & 72.86 & 109.03 & $572 \mathrm{~A}-12-6,83$ & 72.45 & 127.41 & $572 \mathrm{C}-15-5,31$ & 82.11 \\
\hline 101.73 & $572 \mathrm{~A}-12-1,103$ & 65.66 & 109.13 & $572 \mathrm{~A}-12-6,93$ & 68.50 & 127.50 & $572 \mathrm{C}-15-5,40$ & 81.79 \\
\hline 101.83 & $572 \mathrm{~A}-12-1,113$ & 65.10 & 109.23 & $572 \mathrm{~A}-12-6,103$ & 76.95 & 127.62 & $572 \mathrm{C}-15-5,52$ & 83.54 \\
\hline 101.93 & $572 \mathrm{~A}-12-1,123$ & 71.75 & 109.33 & $572 \mathrm{~A}-12-6,113$ & 76.38 & 127.70 & $572 \mathrm{C}-15-5,60$ & 82.20 \\
\hline 102.03 & $572 \mathrm{~A}-12-1,133$ & 70.42 & 109.43 & $572 \mathrm{~A}-12-6,123$ & 73.80 & 127.81 & $572 \mathrm{C}-15-5,71$ & 78.02 \\
\hline 102.13 & $572 \mathrm{~A}-12-1,143$ & 69.53 & 109.43 & $572 \mathrm{~A}-12-6,123$ & 73.80 & 127.90 & $572 \mathrm{C}-15-5,80$ & 81.19 \\
\hline 102.33 & $572 \mathrm{~A}-12-2,13$ & 63.99 & 109.53 & $572 \mathrm{~A}-12-6,133$ & 79.23 & 128.01 & $572 \mathrm{C}-15-5,91$ & 74.63 \\
\hline 102.43 & $572 \mathrm{~A}-12-2,23$ & 67.65 & 109.63 & $572 \mathrm{~A}-12-6,143$ & 79.64 & 128.10 & $572 \mathrm{C}-15-5,100$ & 80.24 \\
\hline 102.53 & $572 \mathrm{~A}-12-2,33$ & 66.21 & 109.73 & $572 \mathrm{~A}-12-7,3$ & 62.56 & 128.21 & $572 \mathrm{C}-15-5,111$ & 80.99 \\
\hline 102.63 & $572 \mathrm{~A}-12-2,43$ & 68.43 & & & & 128.30 & $572 \mathrm{C}-15-5,120$ & 77.52 \\
\hline 102.73 & $572 A-12-2,53$ & 68.43 & 121.10 & $572 \mathrm{C}-15-1,0$ & 75.03 & 128.42 & $572 \mathrm{C}-15-5,132$ & 79.14 \\
\hline 102.83 & $572 A-12-2,63$ & 67.32 & 121.21 & $572 C-15-1,11$ & 83.10 & 128.50 & $572 \mathrm{C}-15-5,140$ & 71.94 \\
\hline 103.23 & $572 \mathrm{~A}-12-2,103$ & 68.98 & 121.30 & $572 \mathrm{C}-15-1,20$ & 76.73 & 128.60 & $572 \mathrm{C}-15-5,150$ & 71.75 \\
\hline 103.43 & $572 \mathrm{~A}-12-2,123$ & 62.88 & 121.41 & $572 \mathrm{C}-15-1,31$ & 65.17 & 128.71 & $572 \mathrm{C}-15-6,11$ & 79.81 \\
\hline 103.53 & $572 \mathrm{~A}-12-2,133$ & 64.88 & 121.50 & $572 \mathrm{C}-15-1,40$ & 50.88 & 128.80 & $572 \mathrm{C}-15-6,20$ & 78.17 \\
\hline 103.63 & $572 \mathrm{~A}-12-2,143$ & 71.20 & 121.70 & $572 C-15-1,60$ & 64.23 & 128.91 & $572 \mathrm{C}-15-6,31$ & 80.93 \\
\hline 103.83 & $572 \mathrm{~A}-12-3,13$ & 78.40 & 121.81 & $572 \mathrm{C}-15-1,71$ & 75.64 & 128.91 & $572 \mathrm{C}-15-6,31$ & 80.93 \\
\hline 103.93 & $572 A-12-3,23$ & 76.74 & 121.90 & $572 \mathrm{C}-15-1,80$ & 72.70 & 129.00 & $572 \mathrm{C}-15-6,40$ & 82.34 \\
\hline 104.03 & $572 \mathrm{~A}-12-3,33$ & 78.40 & 122.01 & $572 \mathrm{C}-15-1,91$ & 73.07 & 129.12 & $572 \mathrm{C}-15-6,52$ & 84.81 \\
\hline 104.03 & $572 A-12-3,33$ & 77.29 & 122.10 & $572 \mathrm{C}-15-1,100$ & 72.32 & 129.20 & $572 \mathrm{C}-15-6,60$ & 81.57 \\
\hline 104.13 & $572 \mathrm{~A}-12-3,43$ & 79.51 & 122.21 & $572 \mathrm{C}-15-1,111$ & 71.39 & 129.24 & $572 C-15-6,64$ & 75.98 \\
\hline 104.33 & $572 A-12-3,63$ & 71.20 & 122.30 & $572 \mathrm{C}-15-1,120$ & 72.18 & 129.31 & $572 \mathrm{C}-15-6,71$ & 78.33 \\
\hline 104.40 & $572 \mathrm{~A}-12-3,70$ & 70.64 & 122.42 & $572 \mathrm{C}-15-1,132$ & 77.47 & 129.35 & $572 \mathrm{C}-15-6,75$ & 74.28 \\
\hline 104.53 & $572 A-12-3,83$ & 68.98 & 122.50 & $572 \mathrm{C}-15-1,140$ & 81.96 & 129.40 & $572 C-15-6,80$ & 81.46 \\
\hline 104.63 & $572 \mathrm{~A}-12-3,93$ & 65.66 & 122.60 & $572 \mathrm{C}-15-2,0$ & 82.25 & 129.44 & $572 \mathrm{C}-15-6,84$ & 71.81 \\
\hline 104.73 & $572 \mathrm{~A}-12-3,103$ & 75.08 & 122.71 & $572 \mathrm{C}-15-2,11$ & 81.07 & 129.51 & $572 \mathrm{C}-15-6,91$ & 77.05 \\
\hline 104.83 & $572 \mathrm{~A}-12-3,113$ & 80.06 & 122.80 & $572 C-15-2,20$ & 86.03 & 129.55 & $572 \mathrm{C}-15-6,95$ & 74.32 \\
\hline 104.93 & $572 \mathrm{~A}-12-3,123$ & 77.29 & 122.91 & $572 \mathrm{C}-15-2,31$ & 79.65 & 129.60 & $572 \mathrm{C}-15-6,100$ & 74.76 \\
\hline 105.03 & $572 \mathrm{~A}-12-3,133$ & 77.29 & 123.00 & $572 \mathrm{C}-15-2,40$ & 81.53 & 129.65 & $572 \mathrm{C}-15-6,105$ & 71.61 \\
\hline 105.13 & $572 \mathrm{~A}-12-3,143$ & 75.08 & 123.12 & $572 \mathrm{C}-15-2,52$ & 75.37 & 129.71 & $572 \mathrm{C}-15-6,111$ & 76.45 \\
\hline 105.23 & $572 \mathrm{~A}-12-4,3$ & 66.21 & 123.20 & $572 C-15-2,60$ & 73.19 & 129.74 & $572 \mathrm{C}-15-6,114$ & 80.73 \\
\hline 105.33 & $572 \mathrm{~A}-12-4,13$ & 60.67 & 123.31 & $572 \mathrm{C}-15-2,71$ & 75.03 & 129.80 & $572 \mathrm{C}-15-6,120$ & 74.41 \\
\hline 105.43 & $572 \mathrm{~A}-12-4,23$ & 68.98 & 123.40 & $572 \mathrm{C}-15-2,80$ & 77.66 & 129.85 & $572 \mathrm{C}-15-6,125$ & 81.68 \\
\hline 105.53 & $572 \mathrm{~A}-12-4,33$ & 72.86 & 123.51 & $572 \mathrm{C}-15-2,91$ & 73.77 & 129.92 & $572 \mathrm{C}-15-6,132$ & 77.54 \\
\hline 105.63 & $572 \mathrm{~A}-12-4,43$ & 80.62 & 123.60 & $572 C-15-2,100$ & 66.40 & 129.94 & $572 \mathrm{C}-15-6,134$ & 74.54 \\
\hline 105.73 & $572 A-12-4,53$ & 73.97 & 123.71 & $572 \mathrm{C}-15-2,111$ & 66.24 & 130.00 & $572 \mathrm{C}-15-6,140$ & 77.02 \\
\hline 105.83 & $572 \mathrm{~A}-12-4,63$ & 76.19 & 123.80 & $572 \mathrm{C}-15-2,120$ & 64.96 & 130.04 & $572 \mathrm{C}-15-6,144$ & 72.88 \\
\hline 105.90 & $572 A-12-4,70$ & 76.19 & 123.92 & $572 \mathrm{C}-15-2,132$ & 67.47 & 130.15 & $572 \mathrm{C}-15-7,5$ & 64.81 \\
\hline 106.03 & $572 \mathrm{~A}-12-4,83$ & 66.21 & 124.00 & $572 \mathrm{C}-15-2,140$ & 66.04 & 130.24 & $572 \mathrm{C}-15-7,14$ & 74.76 \\
\hline 106.13 & $572 \mathrm{~A}-12-4,93$ & 67.65 & 124.10 & $572 \mathrm{C}-15-3,0$ & 62.18 & 130.36 & $572 \mathrm{C}-15-7,26$ & 74.32 \\
\hline 106.23 & $572 \mathrm{~A}-12-4,103$ & 62.33 & 124.21 & $572 \mathrm{C}-15-3,11$ & 59.80 & 130.44 & $572 \mathrm{C}-15-7,34$ & 72.69 \\
\hline 106.33 & $572 \mathrm{~A}-12-4,113$ & 63.99 & 124.30 & $572 \mathrm{C}-15-3,20$ & 58.34 & 130.55 & $572 \mathrm{C}-15-7,45$ & 76.58 \\
\hline 106.43 & $572 \mathrm{~A}-12-4,123$ & 63.44 & 124.41 & $572 \mathrm{C}-15-3,31$ & 59.07 & 130.65 & $572 \mathrm{C}-15-7,55$ & 76.68 \\
\hline 106.53 & $572 \mathrm{~A}-12-4,133$ & 71.75 & 124.50 & $572 \mathrm{C}-15-3,40$ & 64.37 & 131.00 & $572 \mathrm{C}-16-1,30$ & 73.92 \\
\hline 106.63 & $572 \mathrm{~A}-12-4,143$ & 74.52 & 124.70 & $572 \mathrm{C}-15-3,60$ & 72.00 & 131.10 & $572 \mathrm{C}-16-1,40$ & 72.82 \\
\hline 106.73 & $572 \mathrm{~A}-12-5,3$ & 72.18 & 124.81 & $572 \mathrm{C}-15-3,71$ & 74.56 & 131.20 & $572 \mathrm{C}-16-1,50$ & 71.18 \\
\hline 106.83 & $572 \mathrm{~A}-12-5,13$ & 67.33 & 124.90 & $572 \mathrm{C}-15-3,80$ & 75.66 & 131.40 & $572 \mathrm{C}-16-1,70$ & 72.33 \\
\hline 106.93 & $572 \mathrm{~A}-12-5,23$ & 56.89 & 125.01 & $572 \mathrm{C}-15-3,91$ & 70.53 & 131.60 & $572 \mathrm{C}-16-1,90$ & 73.32 \\
\hline 107.03 & $572 A-12-5,33$ & 58.32 & 125.10 & $572 \mathrm{C}-15-3,100$ & 78.54 & 131.70 & $572 \mathrm{C}-16-1,100$ & 75.16 \\
\hline 107.13 & $572 \mathrm{~A}-12-5,43$ & 63.98 & 125.21 & $572 \mathrm{C}-15-3,111$ & 78.04 & 131.82 & $572 \mathrm{C}-16-1,112$ & 74.29 \\
\hline 107.23 & $572 A-12-5,53$ & 78.94 & 125.30 & $572 \mathrm{C}-15-3,120$ & 74.63 & 131.90 & $572 \mathrm{C}-16-1,120$ & 73.01 \\
\hline 107.33 & $572 \mathrm{~A}-12-5,63$ & 70.45 & 125.42 & $572 \mathrm{C}-15-3,132$ & 68.03 & 132.01 & $572 \mathrm{C}-16-1,131$ & 69.70 \\
\hline 107.40 & $572 A-12-5,70$ & 64.72 & 125.50 & $572 \mathrm{C}-15-3,140$ & 79.01 & 132.10 & $572 \mathrm{C}-16-1,140$ & 69.74 \\
\hline 107.53 & $572 \mathrm{~A}-12-5,83$ & 67.66 & 125.60 & $572 \mathrm{C}-15-4,0$ & 80.54 & 132.20 & $572 \mathrm{C}-16-2,0$ & 66.77 \\
\hline 107.63 & $572 \mathrm{~A}-12-5,93$ & 64.39 & 125.71 & $572 C-15-4,11$ & 81.96 & 132.40 & $572 \mathrm{C}-16-2,20$ & 68.40 \\
\hline 107.73 & $572 \mathrm{~A}-12-5,103$ & 65.79 & 125.80 & $572 C-15-4,20$ & 82.92 & 132.51 & $572 \mathrm{C}-16-2,31$ & 70.64 \\
\hline 107.83 & $572 \mathrm{~A}-12-5,113$ & 47.64 & 126.00 & $572 \mathrm{C}-15-4,40$ & 82.92 & 132.60 & $572 \mathrm{C}-16-2,40$ & 71.85 \\
\hline 107.93 & $572 \mathrm{~A}-12-5,123$ & 69.05 & 126.12 & $572 C-15-4,52$ & 80.15 & 132.70 & $572 \mathrm{C}-16-2,50$ & 69.38 \\
\hline 108.03 & $572 \mathrm{~A}-12-5,133$ & 70.52 & 126.20 & $572 \mathrm{C}-15-4,60$ & 75.11 & 132.80 & $572 \mathrm{C}-16-2,60$ & 69.55 \\
\hline 108.13 & $572 \mathrm{~A}-12-5,143$ & 66.51 & 126.31 & $572 C-15-4,71$ & 85.12 & 132.80 & $572 \mathrm{C}-16-2,60$ & 73.27 \\
\hline 108.23 & $572 A-12-6,3$ & 63.07 & 126.40 & $572 \mathrm{C}-15-4,80$ & 78.04 & 132.90 & $572 \mathrm{C}-16-2,70$ & 64.58 \\
\hline 108.33 & $572 A-12-6,13$ & 67.01 & 126.51 & $572 \mathrm{C}-15-4,91$ & 88.18 & 133.00 & $572 \mathrm{C}-16-2,80$ & 64.45 \\
\hline 108.43 & $572 A-12-6,23$ & 67.19 & 126.60 & $572 C-15-4,100$ & 80.15 & 133.10 & $572 \mathrm{C}-16-2,90$ & 63.02 \\
\hline 108.53 & $572 \mathrm{~A}-12-6,33$ & 79.84 & 126.71 & $572 \mathrm{C}-15-4,111$ & 78.70 & 133.20 & $572 C-16-2,100$ & 73.93 \\
\hline 108.63 & $572 A-12-6,43$ & 80.92 & 126.92 & $572 \mathrm{C}-15-4,132$ & 71.58 & 133.20 & $572 \mathrm{C}-16-2,100$ & 81.77 \\
\hline 108.73 & $572 A-12-6,53$ & 79.16 & 127.00 & $572 C-15-4,140$ & 78.30 & 133.40 & $572 \mathrm{C}-16-2,120$ & 53.89 \\
\hline 108.73 & $572 A-12-6,53$ & 72.41 & 127.10 & $572 \mathrm{C}-15-5,0$ & 78.21 & 133.51 & $572 \mathrm{C}-16-2,131$ & 87.24 \\
\hline
\end{tabular}




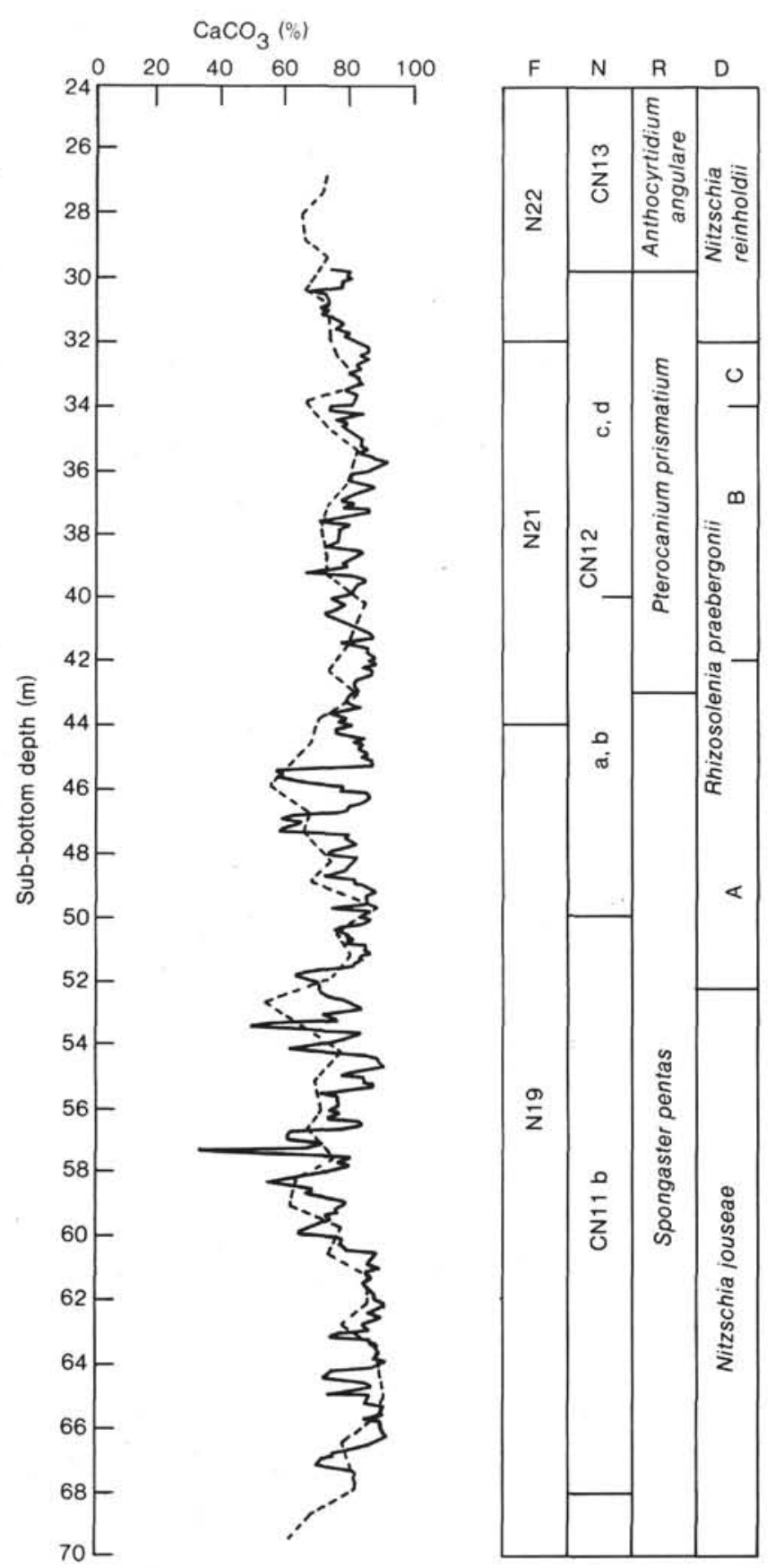

Figure 1. Calcium carbonate versus sub-bottom depth $(27$ to $70 \mathrm{~m})$ in HPC Site 572. Detailed, high resolution carbonate data from Hole $572 \mathrm{C}$ are shown as solid line. Dashed line shows shipboard analyses from Hole 572A. Biostratigraphy is from Site 572 chapter (this volume). $\mathrm{F}=$ planktonic foraminiferal zonation; $\mathrm{N}=$ calcareous nannofossil zonation; $\mathrm{R}=$ radiolarian zonation; $\mathrm{D}=$ diatom zonation.

$79 \% \mathrm{CaCO}_{3}$, with the standard deviations of the intervals ranging from 6.6 to $9.2 \%$ carbonate. (The interval from 30 to $68 \mathrm{~m}$ was divided into two parts, with the number of data points in each approximately equal to the other depth intervals sampled.) Differences between the extreme values of the mean and variance are significant only at the $10 \%$ level (Table 3 ). The average wave-

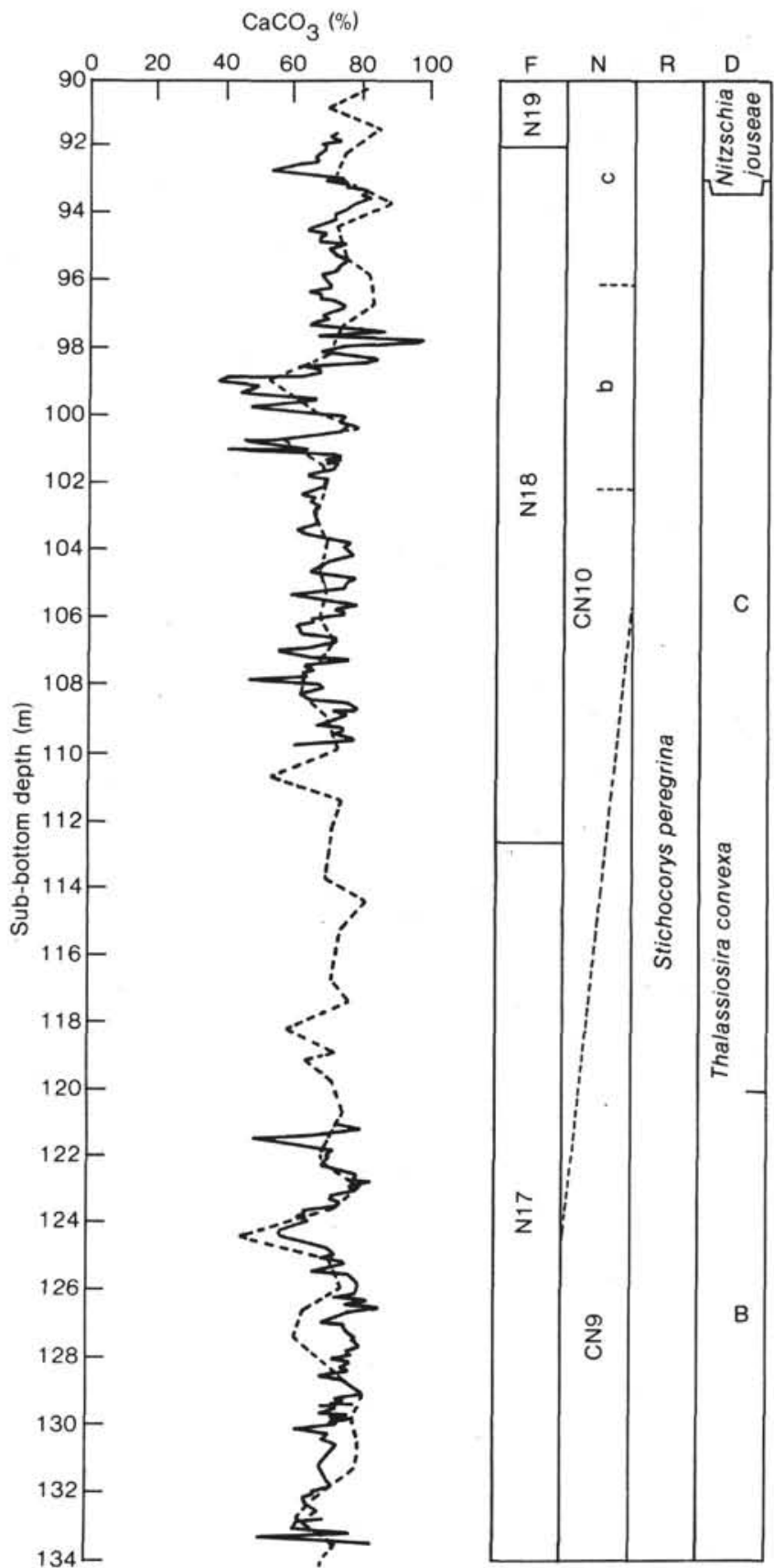

Figure 2. Calcium carbonate versus sub-bottom depth ( 90 to $134 \mathrm{~m}$ ) in HPC Site 572. Detailed high resolution carbonate data from Hole 572A ( 92 to $110 \mathrm{~m})$ and Hole $572 \mathrm{C}(121$ to $134 \mathrm{~m})$ are shown as solid line. Dashed line shows shipboard analyses from Hole 572A. Biostratigraphy plotted from Site 572 chapter (this volume). F, N, $\mathrm{R}, \mathrm{D}$ as defined in Fig. 1.

length of variations in all sections is on the order of 20 to 50 samples per cycle, with secondary fluctuations on the order of 10 samples per cycle (e.g., Fig. 3B).

Although the depth series are remarkably similar, there are significant differences when the data are plotted against age. In Figure 4 the carbonate data are plotted versus ages determined from the sedimentation rates for Site 572 (Table 1). The differences in the frequency of 

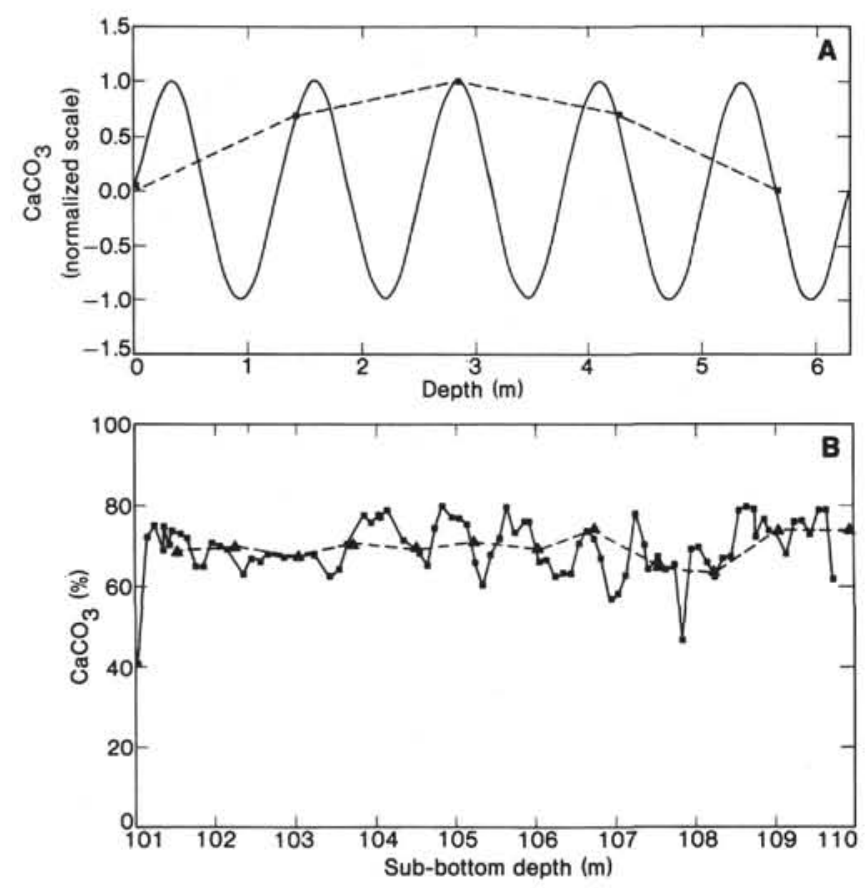

Figure 3. Effects of sample aliasing. A. The effect of sampling a pure sine function with a wavelength of 6 units at a sampling interval of 7 units (1.166 wavelengths). The observed signal is a sine function of 36 units. B. Aliasing of the variations in carbonate as measured by shipboard sampling at $75-\mathrm{cm}$ intervals (dashed line) versus detailed $10-\mathrm{cm}$ interval sampling (solid line). Data points are indicated by squares (detailed data) and triangles (shipboard data).

Table 3. General statistics for high resolution calcium carbonate records from Site 572.

\begin{tabular}{ccccccc}
\hline Hole & $\begin{array}{c}\text { Depth } \\
\text { interval } \\
(\mathrm{m})\end{array}$ & $\begin{array}{c}\text { Time } \\
\text { interval } \\
(\mathrm{Ma})\end{array}$ & $\begin{array}{c}\text { Number } \\
\text { of } \\
\text { samples }\end{array}$ & $\begin{array}{c}\text { Mean } \\
\text { value } \\
\left(\% \mathrm{CaCO}_{3}\right)\end{array}$ & $\begin{array}{c}\text { Variance } \\
(\% 2)\end{array}$ & $\begin{array}{c}\text { Range } \\
\left(\% \mathrm{CaCO}_{3}\right)\end{array}$ \\
\hline $572 \mathrm{C}$ & $30-50$ & $2-3$ & 178 & 75 & 44 & 35 \\
$572 \mathrm{C}$ & $50-90$ & $3-4$ & 180 & 79 & 85 & 59 \\
$572 \mathrm{~A}$ & $92-110$ & $4.7-5$ & 178 & 69 & 75 & 61 \\
$572 \mathrm{C}$ & $121-134$ & $5.2-5.6$ & 141 & 75 & 45 & 37 \\
\hline
\end{tabular}

carbonate variations are striking when the intervals from 4.7 to $5.0 \mathrm{Ma}$ and from 2 to $4 \mathrm{Ma}$ are compared (Fig. 4). (The potential of aliasing of the 2-to-4-Ma record will be discussed later.) These differences are best quantified by considering the frequency domain characteristics. In Figure 5 the variance spectra for the intervals 2 to $3 \mathrm{Ma}$ and 4.7 to $5 \mathrm{Ma}$ are shown. The results of analyses of all four intervals represented in Table 3 are summarized in Table 4. The sample resolution in the 2-to-3-Ma interval is on the order of 6000 to $7000 \mathrm{yr}$., so the highest frequency resolved is equivalent to a 12,000 -to-14,000-yr. period. In this interval ( 2 to $3 \mathrm{Ma}$ ), the spectrum is dominated by three spectral components centered at periods of about $300,000,67,000$, and 35,000 yr. (Fig. 5A). Analysis of both the 2-to-3- and 3-to-4-Ma intervals shows that the amplitudes of the carbonate variation associated with these components (where amplitude is defined as $2 \times$ variance $^{1 / 2}$ associated with the spectral
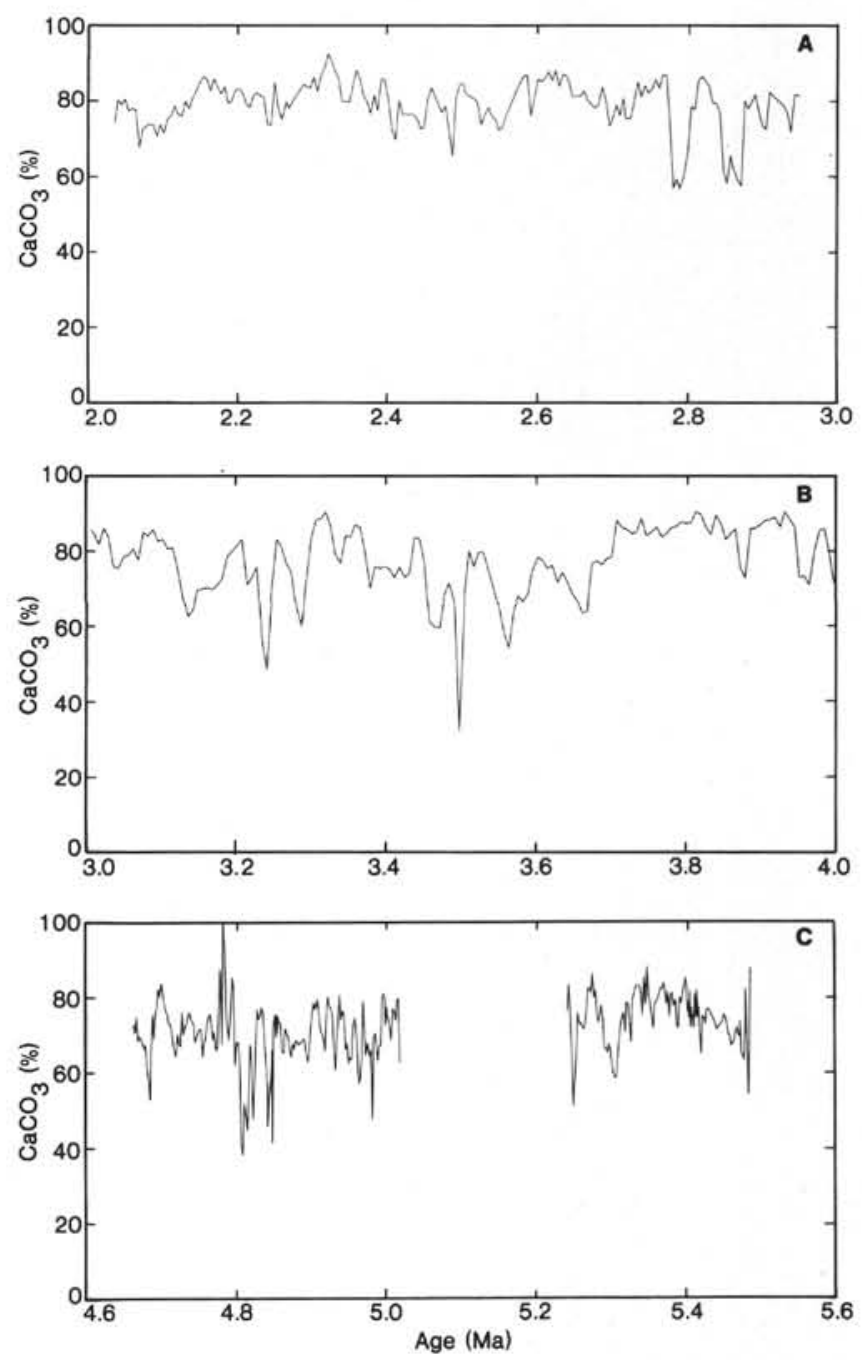

Figure 4. Detailed carbonate data from Site 572 plotted versus age. Age estimates from Site 572 chapter (this volume).

component) are 8 to $12 \%, 7.5$ to $9 \%$, and 2 to $5 \%$, respectively.

In the interval from 4.7 to $5.6 \mathrm{Ma}$ the spectrum shows a significant amount of variance associated with very high frequencies (Fig. 5B). From 12 to $20 \%$ of the total variance in the intervals 4.7 to 5 and 5.2 to $5.6 \mathrm{Ma}$ is found at frequencies equivalent to 16,000 -yr. periods. The dominant spectral component, containing 50 to $60 \%$ of the variance, centers at periods of about 40,000 to $50,000 \mathrm{yr}$. In these late Miocene records, the resolution of long periods is hindered by the limited length of record (about 400,000 yr.). However, the fact that the spectra do not show a continual increase in variance with decreasing frequency of periods over 40,000 yr. suggests that long periods are not major contributors to the variance of the intervals analyzed.

To determine whether the differences between these intervals represent real changes in the variability of the carbonate system of the latest Miocene and Pliocene, we must consider a number of problems. As mentioned above, sample aliasing may be important, because the 


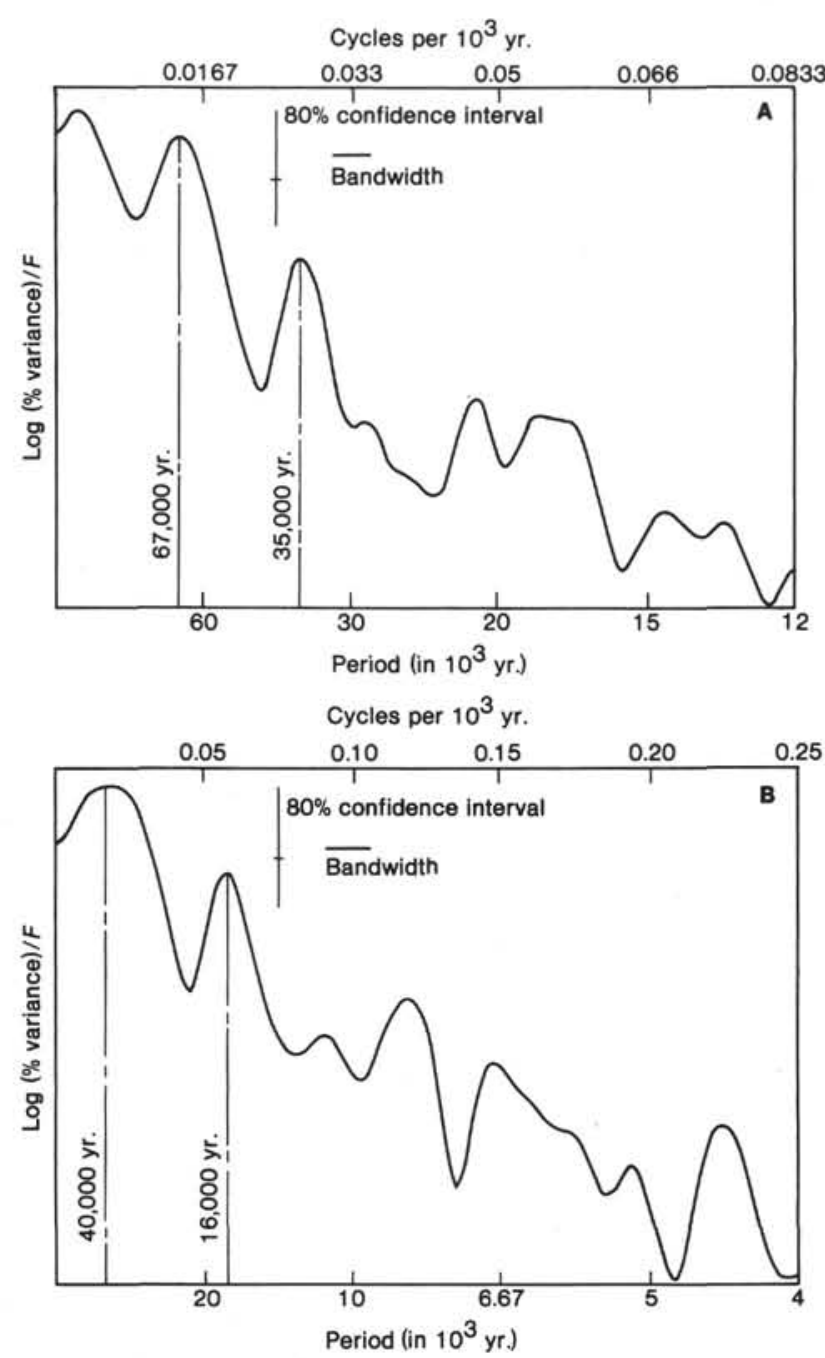

Figure 5. Variance spectra from Site 572 carbonate data. Spectra are plotted on a linear frequency scale and a log variance scale. Confidence interval is for the $80 \%$ level; horizontal bar gives the bandwidth of spectral calculations. Frequencies are in cycles per $10^{3} \mathrm{yr}$. and periods in $10^{3} \mathrm{yr}$. A. Spectra for 2-to-3-Ma interval, Hole 572C (data shown in Fig. 4A). B. Spectra for 4.7-to-5-Ma interval, Hole 572A (data shown in Fig. 4C).

Table 4. Summary of spectral results for detailed calcium carbonate time series from Site 572 .

\begin{tabular}{cccccc}
\hline & \multicolumn{5}{c}{$\begin{array}{c}\text { Variance with frequency bands } \\
\text { (1033-yr. periods) }\end{array}$} \\
\cline { 2 - 6 } $\begin{array}{c}\text { Interval } \\
\text { (Ma) } \\
{[\mathrm{m}]}\end{array}$ & $\infty-142$ & $142-42$ & $42-30$ & Total & $\begin{array}{c}\text { Sample } \\
\text { interval } \\
\text { (yr.) }\end{array}$ \\
\hline $\begin{array}{c}2-3 \\
{[30-50]} \\
3-4 \\
{[50-90]}\end{array}$ & 15 & 14 & 2 & 35 & 6000 \\
& 37 & 21 & 6 & 71 & 6000 \\
& $142-23$ & $23-12.5$ & $12.5-7$ & Total & $\begin{array}{c}\text { Sample } \\
\text { (yr.) }\end{array}$ \\
\cline { 2 - 6 } & 34 & 10 & 6 & 62 & 2000 \\
$\begin{array}{c}4.7-5 \\
{[92-110]} \\
5.2-5.6 \\
{[121-134]}\end{array}$ & 20 & 8 & 3 & 41 & 2000 \\
\hline
\end{tabular}

sampling resolution of the Site 572 data varies by a factor of 3 . Theoretically, the sample spacing of $6000 \mathrm{yr}$. in the interval from 2 to $4 \mathrm{Ma}$ should resolve periods on the order of 16,000 yr. Since no evidence of important periods shorter than 35,000 yr. exists in the Pliocene records, in terms of either significant spectral peaks or in the amount of variance accounted for by high frequencies, it is unlikely that aliasing is an important problem in the Pliocene section.

To verify the absence of sample aliasing in the lower sedimentation rate intervals of Site 572, additional samples were taken at $3-\mathrm{cm}$ intervals to provide a 2000 -yr. resolution. These additional samples (two samples taken between the original $10-\mathrm{cm}$ sample spacing) were taken in the intervals from 30.5 to 33.0 and 35 to $36.5 \mathrm{~m}$ subbottom. The carbonate concentrations measured (using the modified vacuum-gasometric system) in these new samples are very close to the values expected from linear interpolation between the $10-\mathrm{cm}$ samples (Fig. 6), especially for the intervals from 32 to 33 and 35 to $36.5 \mathrm{~m}$. In the interval between 30.5 and $32 \mathrm{~m}$ sub-bottom, the additional samples show slightly more variability than the original $10-\mathrm{cm}$ samples. However, comparison of the means and variance of these intervals estimated from the $10-\mathrm{cm}$ and $3-\mathrm{cm}$ sample sets shows no significant differences. It should be noted that the similarity between the samples at $10-\mathrm{cm}$ intervals and the data set sampled at 3-cm intervals, reflects, in part, the mixing of pelagic sediments by benthic organisms.

In addition to sample aliasing as a possible cause for an apparent difference between the Miocene and Pliocene records, there may be significant error in the mean sedimentation rates used to determine the time scales for these records. Magnetic stratigraphy is not available for Site 572 , and sedimentation rate estimates were made by using radiolarian and diatom datums that have been correlated to the geomagnetic time scale by a number of investigators (see Barron et al., this volume). Stratigraphic analysis of all sites drilled on Leg 85 suggests that correlations to within a few meters are possible throughout the Pliocene and Miocene sections of Sites 573 and 572. Thus, it is possible to correlate accurately the magnetic stratigraphy of Site $\mathbf{5 7 3}$ with the Site $\mathbf{5 7 2}$ data (Pisias et al., this volume; Prell, this volume). Such correlations also support the estimated sedimentation rate estimates used in this study.

If the change in sedimentation rates estimated between the intervals from 68 to $208 \mathrm{~m}$ and 29 to $68 \mathrm{~m}$ subbottom is correct, the mass accumulation rate of calcium carbonate changes from 3.5 to $1 \mathrm{~g} / \mathrm{cm}^{2}$ per $10^{3} \mathrm{yr}$., respectively. Associated with this threefold decrease in mass accumulation rate (Table 1) is a marked decrease in high-frequency variations in carbonate content. Thus, if the carbonate variations are converted to mass accumulation rates, the latest Miocene carbonate record at Site 572 requires not only high-frequency variations of carbonate content but also very high amplitude variations in the total mass of carbonate dissolved or preserved as compared to the Pliocene interval. However, these mass accumulation rate differences may have an effect on the nature of the variability of the carbonate content in a sediment column. 

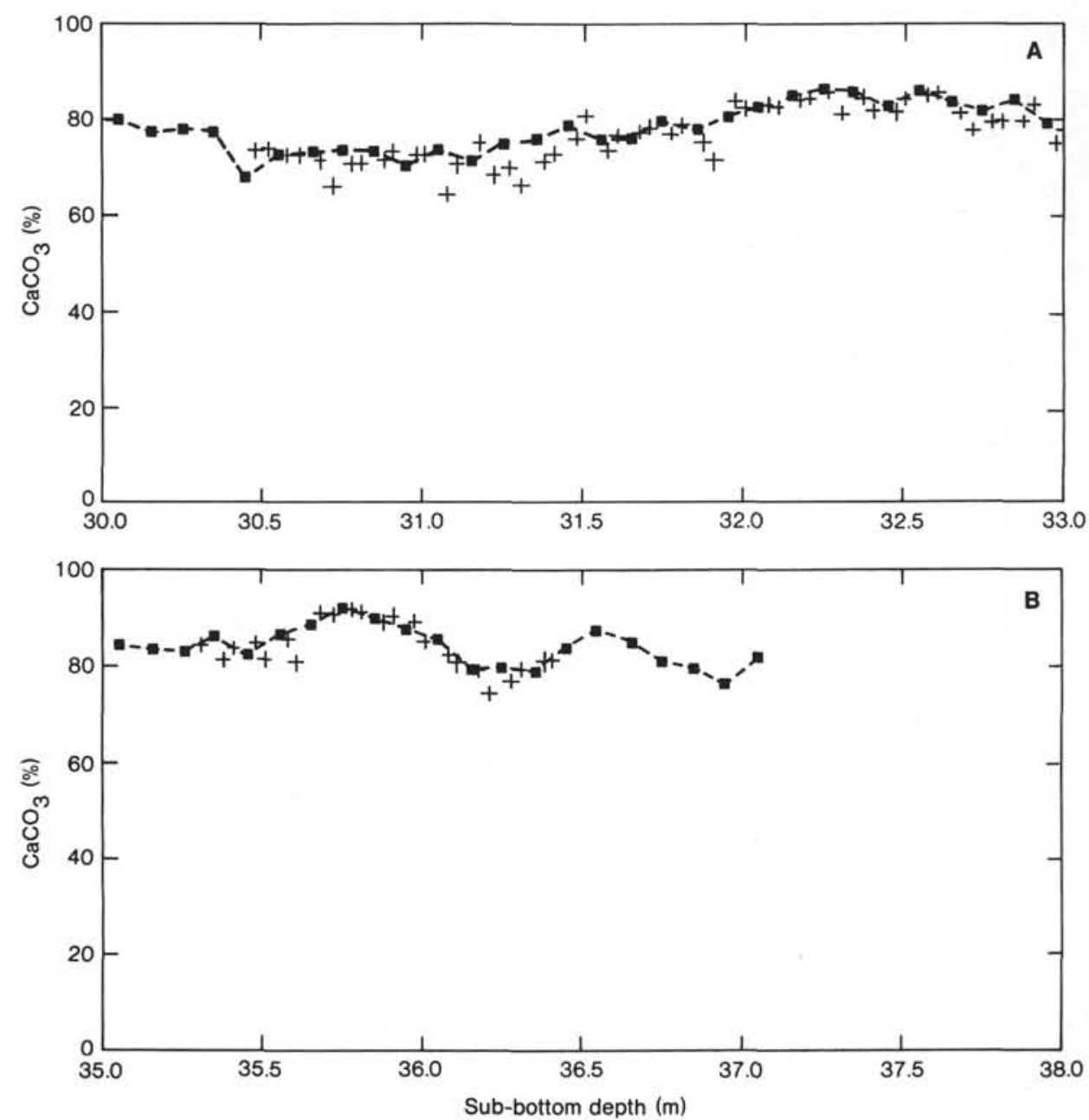

Figure 6. Detailed sampling of Hole $572 \mathrm{C}$ done to determine whether sample aliasing was present. Solid squares represent samples at 10-cm sample spacing, and crosses represent additional samples taken at about 3-cm intervals. Note the close agreement of 3-cm samples and values expected from linear interpolation (dashed line) between the $10-\mathrm{cm}$ samples in the intervals 32 to 33 and 35 to $36.5 \mathrm{~m}$ subbottom. All samples analyzed with high precision carbonate analysis technique described in text. A. Sub-bottom depths 30 to $33 \mathrm{~m}$. B. Sub-bottom depths 35 to $37 \mathrm{~m}$.

\section{Causes of Carbonate Fluctuations-A Mass Accumulation Rate Approach}

A useful review of the hypotheses for variation in calcium carbonate concentrations is given by Volat et al. (1980). The weight percentage of calcium carbonate in pelagic sediments reflects the balance between the rate of input of calcium carbonate to the sediment surface, the rate of carbonate dissolution, and rate of accumulation of non-carbonate diluents. The major noncarbonate sediment at Site $\mathbf{5 7 2}$ is biogenic silica (opal), so the rate of noncarbonate deposition reflects the rate of input versus the rate of dissolution of opal.

The effects of the factors controlling carbonate deposition can be modeled as in Figure 7 (Heath and Culberson, 1970; Dean et al., 1981; Gardner, 1982). In Figure 7 it is assumed that the initial pelagic rain is $85 \%$ carbonate and $15 \%$ noncarbonate. (Note that the assumption of an initial pelagic rain of $85 \%$ would require some dissolution of noncarbonate material at the sediment surface to account for higher $\mathrm{CaCO}_{3}$ contents in some of the Site 572 samples.) Variations in the concentration of calcium carbonate can result from changes in dilution by non- $\mathrm{CaCO}_{3}$ or from changes in the percentage of calcium carbonate dissolved. The percentage of dilution $D$ needed to produce the observed calcium carbonate $C_{\mathrm{f}}$ from the initial carbonate content $C_{\mathrm{i}}$ (Gardner, 1975) is

$$
D=100\left(\left[C_{\mathrm{i}} / C_{\mathrm{f}}\right]-1\right) /\left(1-C_{\mathrm{i}}\right)
$$

The percentage carbonate lost $(L)$ to dissolution needed to produce the observed noncarbonate fraction $N_{\mathrm{f}}$ from the initial fraction $N_{\mathrm{i}}$ is

$$
L=100\left(1-\left[N_{\mathrm{i}} / N_{\mathrm{f}}\right]\right) /\left(1-N_{\mathrm{i}}\right)
$$

Analyses of late Pleistocene sediments (Volat et al., 1980) and Cenozoic sections (Dean et al., 1981; Dunn and Moore, 1981) suggest that dissolution rather than dilution plays the dominant role in controlling carbonate variations. In the following discussion we infer that dissolution plays an important role in controlling pre- 


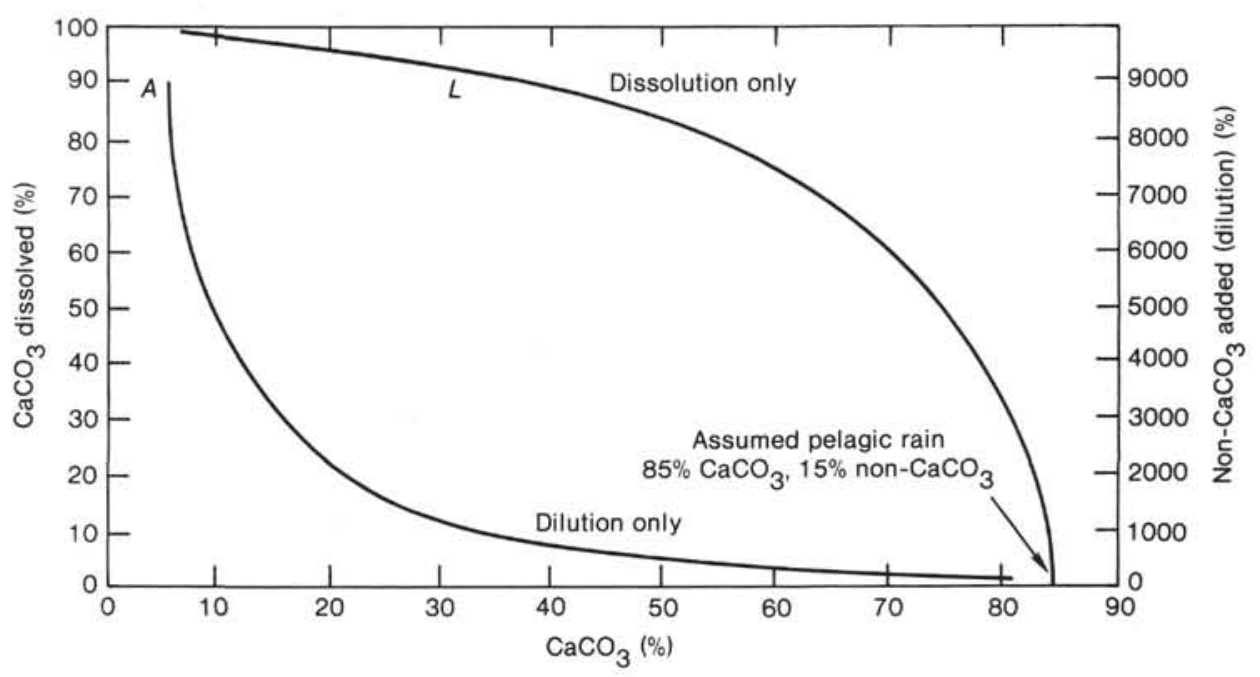

Figure 7. Curves for the loss of carbonate by dissolution $(L)$ and addition of noncarbonate dilutents $(A)$ to an initial carbonate rain of $85 \% \mathrm{CaCO}_{3}$ (after Dean et al., 1981).

Pleistocene carbonate variations in Site 572, but without detailed studies of carbonate preservation we cannot eliminate variations in biogenic silica deposition or preservation as important controls.

As is apparent in Figure 7, changing the observed carbonate content from an initial $85 \%$ to a value of $80 \%$ by dissolution alone requires a minimum of $30 \%$ of the initial carbonate to be dissolved. If we compare two different sediment records, as in the case of the Pliocene and Miocene at Site 572, and calculate the percentage dissolution required to describe the individual records, we have inherently assumed that dissolution is the only process responsible for changes in carbonate content (i.e., there are no changes in supply or dilution by other sediment fractions) and that the system is steadystate over each interval. This implies that for a given change in $\mathrm{CaCO}_{3}$ content, the proportion of $\mathrm{CaCO}_{3}$ that has been dissolved is constant and is independent of the absolute rate of dissolution. For example, the change from 85 to $80 \% \mathrm{CaCO}_{3}$ would require three times the rate of dissolution for a sediment column with a carbonate deposition rate of $3 \mathrm{~g} / \mathrm{cm}^{2}$ per $10^{3} \mathrm{yr}$. as it would for one with a rate of $1 \mathrm{~g} / \mathrm{cm}^{2}$ per $10^{3}$ yr. For the Site 572 data, this assumption of dissolution-controlled variation would suggest that the amplitude of variation in $\mathrm{CaCO}_{3}$ percentages in the uppermost Miocene sediment (which is similar to the Pliocene data) would represent a calcium carbonate dissolution rate of three times that needed to produce the observed carbonate percentage variations in the Pliocene sediment. Thus, not only was the wavelength of carbonate variations much shorter during the Miocene, but the variations in the total mass of carbonate dissolved were significantly higher.

Since the mass of carbonate preserved, or the rate of dissolution, provides more information than $\mathrm{CaCO}_{3}$ percentages, we suggest a different approach to describing changes in carbonate content. It is our belief that changes from the mean concentration of calcium carbonate caused by specific changes in oceanographic processes are best described in terms of changing rates of dissolution rather than simply changing proportions of carbonate preserved at any one location.

An example of an important consequence of this approach is to consider the amplitude of carbonate variations associated with the 67,000-yr. spectral component of the 2-to-3-Ma carbonate record of Site 572 (Fig. 5A). The amplitude of this frequency component is about $10 \% \mathrm{CaCO}_{3}$ in the intervals with mean sediment accumulation rates of $1.00 \mathrm{~g} / \mathrm{cm}^{2}$ per $10^{3} \mathrm{yr}$. and a mean carbonate content of $75 \%$ (Table 1). If we assume that the supply of non- $\mathrm{CaCO}_{3}$ sediment has remained constant, a change in the mass accumulation of $\mathrm{CaCO}_{3}$ of $0.2 \mathrm{~g}$ / $\mathrm{cm}^{2}$ per $10^{3} \mathrm{yr}$. is required to produce the observed $10 \%$ carbonate content signal. The value of $0.2 \mathrm{~g} / \mathrm{cm}^{2}$ per $10^{3} \mathrm{yr}$. is the amplitude of the change in accumulation rate associated with the 67,000 -yr. frequency component and may reflect changes in dissolution as well as production. If the total mass accumulation rate was $3.5 \mathrm{~g} /$ $\mathrm{cm}^{2}$ per $10^{3} \mathrm{yr}$. as in the 5.2-to-5.6-Ma interval, the 0.2 $\mathrm{g} / \mathrm{cm}^{2}$ per $10^{3} \mathrm{yr}$. change in carbonate deposition would produce a carbonate content change of only $4 \%$.

The relationship between the amplitude of the change in the carbonate concentration and the mean mass accumulation rate can be calculated for a given mean carbonate percentage ( $75 \%$ is used in Fig. $8 \mathrm{~A})$. Two values for the net change in carbonate mass accumulation rates $\left(0.2\right.$ and $0.35 \mathrm{~g} / \mathrm{cm}^{2}$ per $10^{3} \mathrm{yr}$.) are shown in Figure 8A. A mean of $75 \%$ was chosen, because this is a typical value for the mean carbonate value of all sites drilled on Leg 85 in the equatorial Pacific. As shown in Figure 8A, for mass accumulation rates of 0.5 to $2.0 \mathrm{~g} / \mathrm{cm}^{2}$ per $10^{3} \mathrm{yr}$. (sedimentation rates on the order of 1 to $3 \mathrm{~cm}$ / $10^{3} \mathrm{yr}$ ), a $0.2 \mathrm{~g} / \mathrm{cm}^{2}$ per $10^{3} \mathrm{yr}$. rate change in carbonate deposition is reflected in carbonate concentration as a variation by a factor of 5 (from 40 to $8 \% \mathrm{CaCO}_{3}$ ).

Thus far we have assumed that changes in carbonate concentrations reflect changes in net carbonate mass accumulation rates (i.e., our supply-dissolution model). If we consider that changes in calcium carbonate content at Site 572 reflect changes in noncarbonate (dilu- 

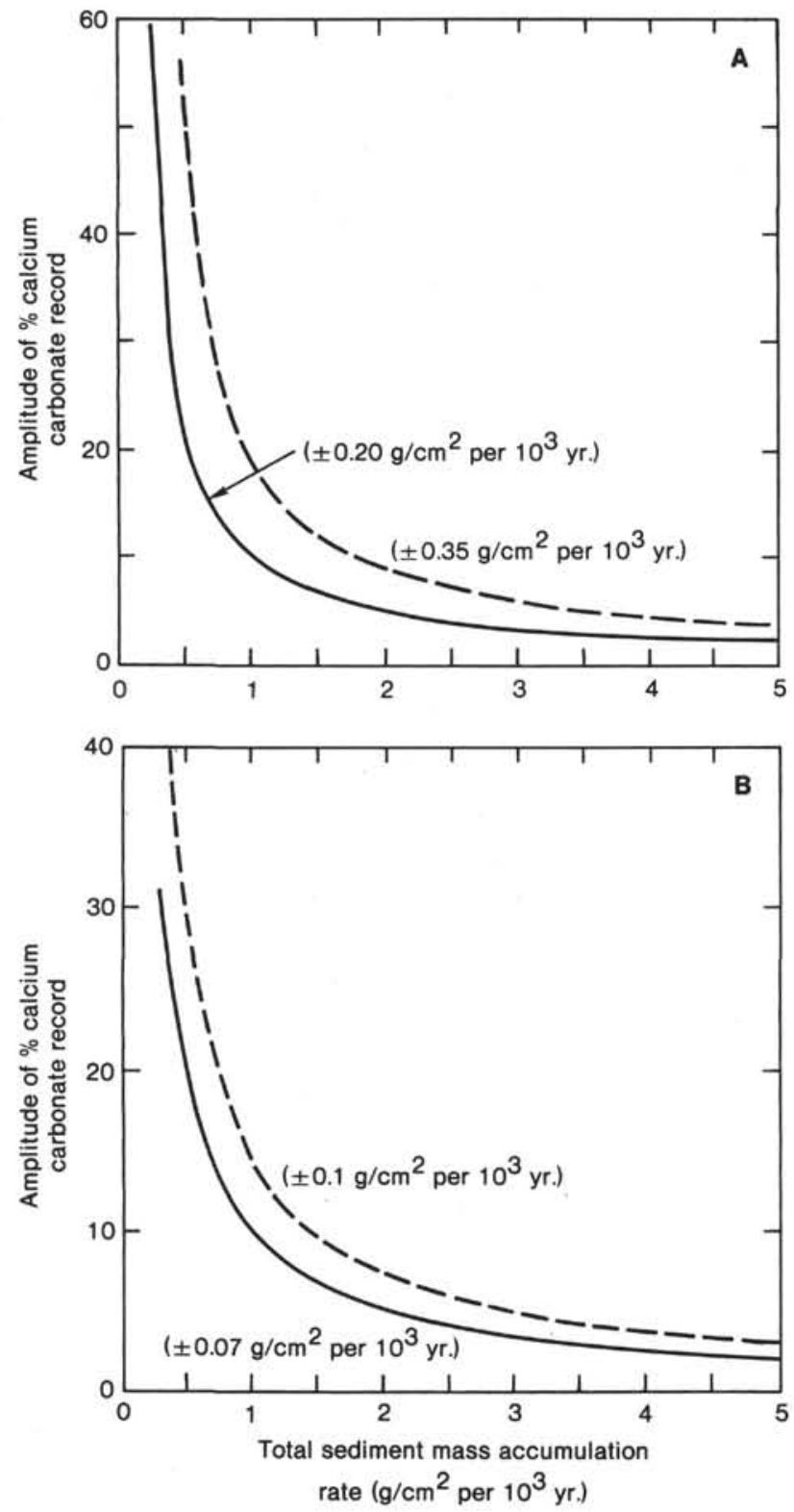

Figure 8. Amplitude of the carbonate concentration record versus total mean accumulation rates for a given response in the net accumulation rate of carbonate or noncarbonate. A. Calculations made assuming a mean carbonate concentration of $75 \%$, a constant noncarbonate flux, and a change in net carbonate deposition of 0.20 and $0.35 \mathrm{~g} / \mathrm{cm}^{2}$ per $10^{3}$. B. Calculations assume a mean carbonate concentration of $75 \%$, a constant carbonate flux, and a change in net noncarbonate accumulation of 0.07 and $0.10 \mathrm{~g} / \mathrm{cm}^{2}$ per $10^{3} \mathrm{yr}$.

tion), we can calculate the change in the mass accumulation necessary to produce carbonate fluctuations. If we use the same starting values (a mean mass accumulation rate of $1 \mathrm{~g} / \mathrm{cm}^{2}$ per $10^{3} \mathrm{yr}$. and a mean carbonate content of $75 \%$ ), a change in net noncarbonate accumulation of $0.07 \mathrm{~g} / \mathrm{cm}^{2}$ per $10^{3} \mathrm{yr}$. is required to produce a $10 \% \mathrm{CaCO}_{3}$ amplitude in the carbonate record. Although this value is less than half of the change needed if only variations in carbonate supply and dissolution are considered, it represents $30 \%$ of the mean noncarbonate accumulation. As shown in Figure 8B, the am- plitude of carbonate variations caused by changes in noncarbonate deposition also varies as a function of total mean accumulation. An increase in the amplitude of variations in carbonate content with decreasing sedimentation rates is produced by variation in noncarbonate accumulation rates.

Without detailed studies of the degree of carbonate dissolution in the sediments from Site $\mathbf{5 7 2}$ or detailed time control to constrain very short-term changes in sedimentation rates, it is difficult to evaluate the ultimate cause of the carbonate content fluctuations in this equatorial Pacific site. However, one possible constraint is supplied by the variance spectrum calculated from these data. If changes in the $\mathrm{CaCO}_{3}$ records reflect changes in carbonate supply and/or dissolution, then we would expect significant changes in the short-term mass accumulation rates. In Figure 9 the percentage change in the total accumulation rate associated with the estimated 0.2 and $0.07 \mathrm{~g} / \mathrm{cm}^{2}$ per $10^{3} \mathrm{yr}$. rate changes in carbonate and noncarbonate accumulation is plotted as a function of mean sedimentation rates. For a mean rate of $1 \mathrm{~g} / \mathrm{cm}^{2}$ per $10^{3} \mathrm{yr}$., changes in noncarbonate deposition would produce a $15 \%$ change in the total accumulation, as opposed to a $40 \%$ change if carbonate deposition is controlling the carbonate concentrations. In Figure 4 the carbonate minima tend to occur over shorter depth intervals relative to maxima. This could reflect reduced sedimentation rates during intervals of decreased carbonate concentration. This type of sedimentation rate distortion would produce harmonics in the variance spectra associated with important periodic components (Holman and Pisias, 1983). In the 2-to-3-Ma interval of the Site 572 data, the secondary spectral peak $(35,000 \mathrm{yr})$ is very close to the predicted harmonic of the 67,000 -yr. com-

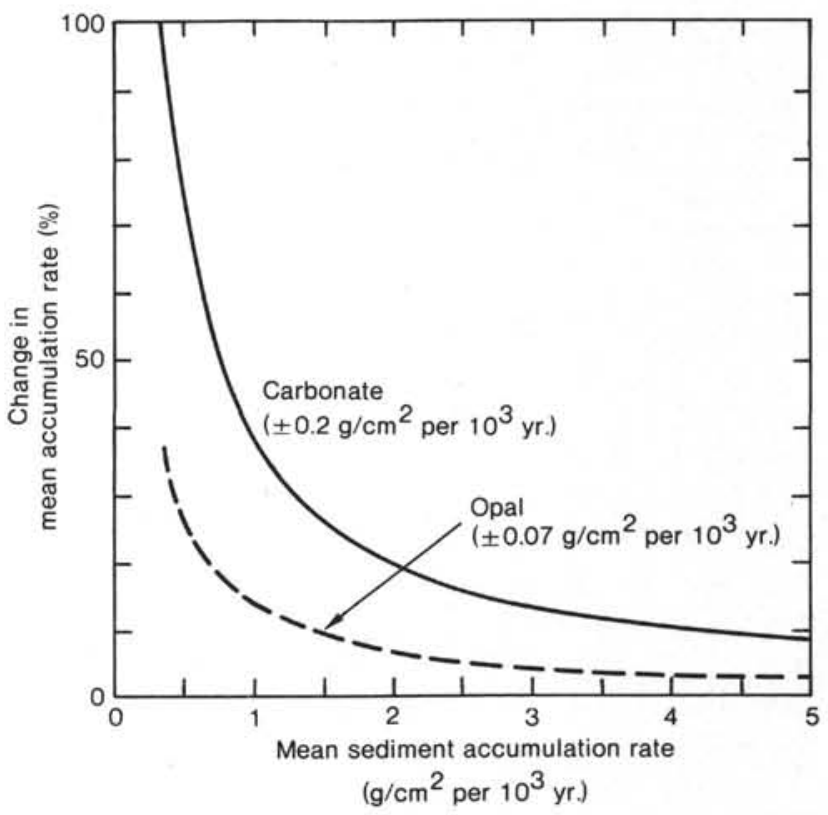

Figure 9. Percentage change in mean accumulation rate versus mean accumulation rate for a $0.07 \mathrm{~g} / \mathrm{cm}^{2}$ per $10^{3} \mathrm{yr}$. change in the noncarbonate accumulation rate (dashed line) and a $0.2 \mathrm{~g} / \mathrm{cm}^{2}$ per $10^{3}$ yr. change in the carbonate accumulation rate (solid line). 
ponent. Further study is necessary to evaluate whether the variance and phase of the 35,000-yr. component is consistent with sedimentation rate changes on the order of $67,000 \mathrm{yr}$.

These calculations of carbonate content changes relative to mass accumulation rates have important implications for the resolution of carbonate records from lower sedimentation rate intervals. As sedimentation rates decrease and/or sampling intervals increase, then aliasing becomes an important problem. If the amplitude of the carbonate variation also tends to increase with decreasing sedimentation rates, the effect of aliasing becomes even more exaggerated.

\section{CONCLUSIONS}

Detailed analysis of calcium carbonate concentrations in the HPC sections of Site 572 shows a significant difference between the nature of carbonate deposition at this equatorial site during the late Miocene and during the Pliocene. Both the degree of variability of carbonate deposition and the total sediment and carbonate accumulation rates were markedly higher in the late Miocene than in the Pliocene intervals. The present data set, however, does not allow us to determine the nature of the transition between these two regimes of carbonate deposition.

Calculations of changes in mass accumulation rates suggest that the nature of a carbonate concentration record is controlled in part by total mass accumulation rates and that small changes in the mass accumulation of noncarbonate material have a significant effect on the carbonate concentration data. Thus, without detailed analysis of the changes in mass accumulation rates or the influence of dissolution, it is difficult to determine the true causes of the carbonate variations observed.

\section{REFERENCES}

Dean, W. E., Gardner, J. V., and Cepek, P., 1981. Tertiary carbonatedissolution cycles on the Sierra Leone Rise, eastern equatorial Atlantic Ocean. Mar. Geol., 39:81-101.

Dunn, D. A., 1980. Revised technique for quantitative calcium carbonate analysis using the "Karbonat-Bombe" and correlations to other quantitative carbonate analysis methods. J. Sediment. Petrol., 50:631-637.

Dunn, D. A., and Moore, T. C., Jr., 1981. Late Miocene-Pliocene (Magnetic Epoch 9-Gilbert Magnetic Epoch) calcium carbonate stratigraphy of the equatorial Pacific. Geol. Soc. Am. Bull., Pt. I, 92:104-107.

Gardner, J. V., 1975. Late Pleistocene carbonate dissolution cycles in the eastern equatorial Atlantic. In Sliter, W. V., Bé, A. W. H., and Berger, W. H. (Eds.), Dissolution of Deep-Sea Carbonates. Spec. Publ. Cushman Found. Foraminiferal Res., 13:129-141.

1982. High-resolution carbonate and organic-carbon stratigraphies for the late Neogene and Quaternary from the eastern equatorial Pacific. In Prell, W. L., Gardner, J. V., et al., Init. Repts. DSDP, 68: Washington (U.S. Govt. Printing Office), 347-364.

Hays, J. D., Saito, T., Opdyke, N. D., and Burckle, L. H., 1969. Pliocene-Pleistocene sediments of the equatorial Pacific: their paleomagnetic, biostratigraphic, and climatic record. Geol. Soc. Am. Bull., 80(8):1481-1514.

Heath, G. R., and Culberson, C., 1970. Calcite: degree of saturation, rate of dissolution and compensation depth in the deep oceans. Geol. Soc. Am. Bull., 81(10):3157-3160.

Holman, R. A., and Pisias, N. G., 1983. Geologic time series: distortion due to periodic variations in sedimentation rates. Trans. Am. Geophys. Union, 64(45):738. (Abstract)

Jones, G. A., and Kaiteris, P., 1983. A vacuum-gasometric technique for rapid and precise analysis of calcium carbonate in sediments and soils. J. Sediment. Petrol., 53(2):655-659.

Moore, T. C., Jr., Pisias, N. G., and Dunn, D. A., 1982. Carbonate time series of the Quaternary and late Miocene sediments in the Pacific Ocean: a spectral comparison. Mar. Geol., 46:217.

Prell, W. L., 1978. Upper Quaternary sediments of the Colombia Basin: spatial and stratigraphic variations. Geol. Soc. Am. Bull., 89(8):1241-1255.

1982. Oxygen and carbon isotope stratigraphy for the Quaternary of Hole 502B: evidence for two modes in isotopic variability. In Prell, W. L., Gardner, J. V., et al., Init. Repts. DSDP, 68: Washington (U.S. Govt. Printing Office), 455-464.

Shackleton, N. J., Backman, J., Zimmerman, H., Kent, D. V., Hall, M. A., Roberts, D. G., Schnitker, D., Baldauf, J. G., Desprairies, A., Homrighausen, R., Huddlestun, P., Keene, J. B., Kaltenback, A. J., Krumsiek, K. A. O., Morton, A. C., Murray, J. W., and Westberg-Smith, J., 1984. Oxygen isotope calibration of the onset of ice-rafting and history of glaciation in the North Atlantic region. Nature, 307:620-623.

Vincent, E., 1981. Neogene carbonate stratigraphy of Hess Rise (central North Pacific) and paleoceanographic implications. In Thiede, J., Vallier, T. L., et al., Init. Repts. DSDP, 62: Washington (U.S. Govt. Printing Office), 571-606.

Volat, J. L., Pastouret, L., and Vergnaud Grazzini, C., 1980. Dissolution and carbonate fluctuations in Pleistocene deep-sea cores: a review. Mar. Geol., 34:1-28.

Woodruff, F., Savin, S. M., and Douglas, R. G., 1981. Miocene stable isotope record: a detailed deep Pacific Ocean study and its paleoclimatic implications. Science, 212:665-668.

Date of Initial Receipt: 28 October 1983

Date of Acceptance: 18 April 1984 